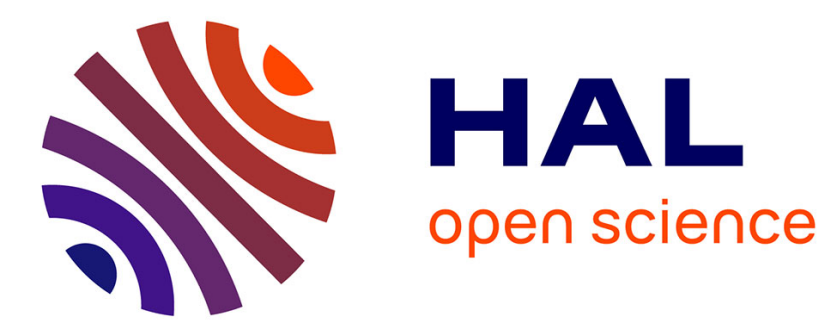

\title{
Asymmetric non-linear forced vibrations of free-edge circular plates. Part II: Experiments
}

Olivier Thomas, Cyril Touzé, Antoine Chaigne

\section{To cite this version:}

Olivier Thomas, Cyril Touzé, Antoine Chaigne. Asymmetric non-linear forced vibrations of free-edge circular plates. Part II: Experiments. Journal of Sound and Vibration, 2003, 265 (5), pp.1075-1101. 10.1016/s0022-460x(02)01564-x . hal-00830696

\section{HAL Id: hal-00830696 \\ https://hal-ensta-paris.archives-ouvertes.fr/hal-00830696}

Submitted on 17 Mar 2016

HAL is a multi-disciplinary open access archive for the deposit and dissemination of scientific research documents, whether they are published or not. The documents may come from teaching and research institutions in France or abroad, or from public or private research centers.
L'archive ouverte pluridisciplinaire HAL, est destinée au dépôt et à la diffusion de documents scientifiques de niveau recherche, publiés ou non, émanant des établissements d'enseignement et de recherche français ou étrangers, des laboratoires publics ou privés. 


\title{
Asymmetric non-linear forced vibrations of free-edge circular plates. Part II: experiments
}

\author{
O. Thomas ${ }^{\mathrm{a}, \mathrm{b}, *}$, C. Touzé ${ }^{\mathrm{b}}$, A. Chaigne ${ }^{\mathrm{b}}$ \\ ${ }^{a}$ ENST, Département TSI, 46 rue Barrault, 75634 Paris Cedex 13, France \\ ${ }^{\mathrm{b}}$ ENSTA - UME, Chemin de la Hunière, 91761 Palaiseau Cedex, France
}

\begin{abstract}
This article is dev oted to an experimental $\mathrm{v}$ alidation of a theoretical model presented in an earlier contribution by the same authors. The non-linear forced vibrations of circular plates, with the excitation frequency close to the natural frequency of an asymmetric mode, are investigated. The experimental set-up, which allows one to perform precise measurements of the vibration amplitudes of the two preferential configurations, is presented. Experimental resonance curves showing the amplitude and the phase of each configuration as functions of the driving frequency are compared to the theoretical ones, leading to a quantitative $\mathrm{v}$ alidation of the predictions giv en by the model. Finally, all the approximations used are systematically discussed, in order to show the scope and relevance of the approach.
\end{abstract}

\section{Introduction}

In an earlier paper [1], (hereafter referred to as Part I), a theoretical model of a circular plate subjected to geometrically non-linear forced vibrations, when the driving frequency is close to the natural frequency of an asymmetric mode of the plate, was derived. This article presents a series of measurements performed on asymmetrically forced circular plates, in order to validate the theoretical developments presented in Part I.

Asymmetric non-linear vibrations of circular plates have received little attention, as opposed to the axisymmetric case, which has been widely treated theoretically and experimentally [2-5]. 
For the asymmetric case, the pioneering work of Tobias et al. seems to be the only one to give a comprehensive study, of both the theoretical and experimental viewpoints [6-8]. But some features were not elucidated at that time, as it is mentioned by the open questions left at the end of Ref. [7]. Moreover, crucial physical parameters such as damping were neglected. More recently, mainly theoretical developments have been performed. Subharmonic oscillations of an asymmetric eigenmode of a circular plate has been studied in Ref. [9]; asymmetric modal coupling has been taken into account in Ref. [10]. In contrast, experimental validations remain seldom seen. Yasuda and Asano show experimental measurements on a rectangular plate in which degenerated modes occur, i.e., one-to-one internally resonant modes [11]. But to the authors' knowledge, only the contribution of Raman and Mote [12], concerned with experiments performed on circular plates subjected to asymmetric vibrations, has been published since the work of Tobias et al.

As the plate is forced with a frequency close to the natural frequency, the deflection of the plate is governed by the corresponding modal shape(s) only. If the mode is asymmetric, two modes, called preferential configurations, with slightly different modal shapes and natural frequencies, are involved in the vibration [1,8]. The experimental set-up made in our laboratory with a brass circular plate is presented. This set-up enabled us to measure the deflections of an antinode of each preferential configuration, so that their respective contributions in the vibration are identified. Experimental resonance curves showing the amplitude and the phase of each configuration as functions of the driving frequency are analyzed. Jump phenomena are exhibited, as well as energy exchanges between the two configurations due to non-linear coupling. This coupling leads to a rotating travelling wave, predicted in Part I and mentioned in Refs. [6-8]. The resonance curves are compared to the ones derived from the model of Part I, leading to a quantitative validation of the predictions of the theory. Finally, all approximations used are systematically discussed.

\section{Experimental details}

In the experiments, a circular plate of outer diameter $2 a=220 \mathrm{~mm}$, thickness $h=1.6 \mathrm{~mm}$, made of brass of density $\rho=7974 \mathrm{~kg} \mathrm{~m}^{-3}$, Young's modulus $E=85 \times 10^{9} \mathrm{~Pa}$ and Poisson ratio $v=0.38$ is used. Three small holes (of diameter $2 \mathrm{~mm}$ ) at the rim allow us to hang up the plate with nylon threads.

\subsection{Apparatus}

The apparatus used during the experiments is shown in Fig. 1 and the references of the different measuring devices are listed in Table 1.

The plate is excited by means of a magnet, glued with beeswax and driven by a coil (Fig. 2). The location of the magnet was chosen to be on a nodal diameter of one preferential configuration, so that it mainly excites the other configuration (Fig. 3). The coil is fed through a power amplifier by an harmonic signal of frequency $\Omega$ generated by a synthetizer. Because this signal is not purely sinusoidal, its harmonics (of frequencies $2 \Omega, 3 \Omega, \ldots$ ) are eliminated by a low-pass filter. 


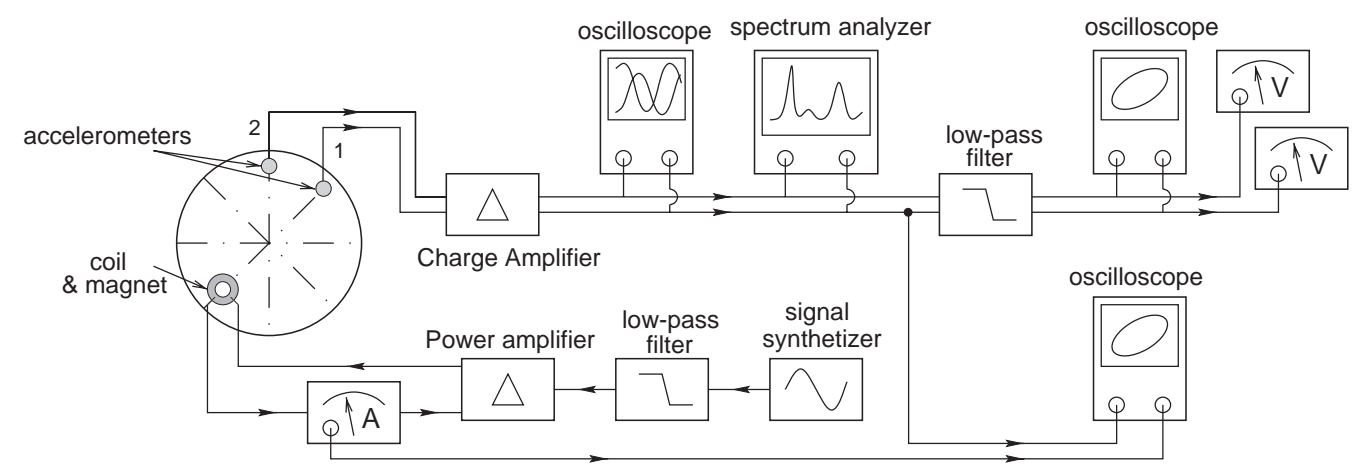

Fig. 1. Experimental apparatus.

Table 1

Devices used during experiments

Accelerometer $1 \& 2$

Charge amplifier

Low-pass filters

Voltmeters

Signal synthetizer

Power amplifier

Ammeter
Bruel \& Kjær

Bruel \& Kjær

Rockland

Philips

Fluke

Crown

Hewlett-Packard
4374

Nexus $^{\mathrm{TM}}$

$1042 \mathrm{~F}$

PM2519

PM5193

Macrotech 2400

3478A

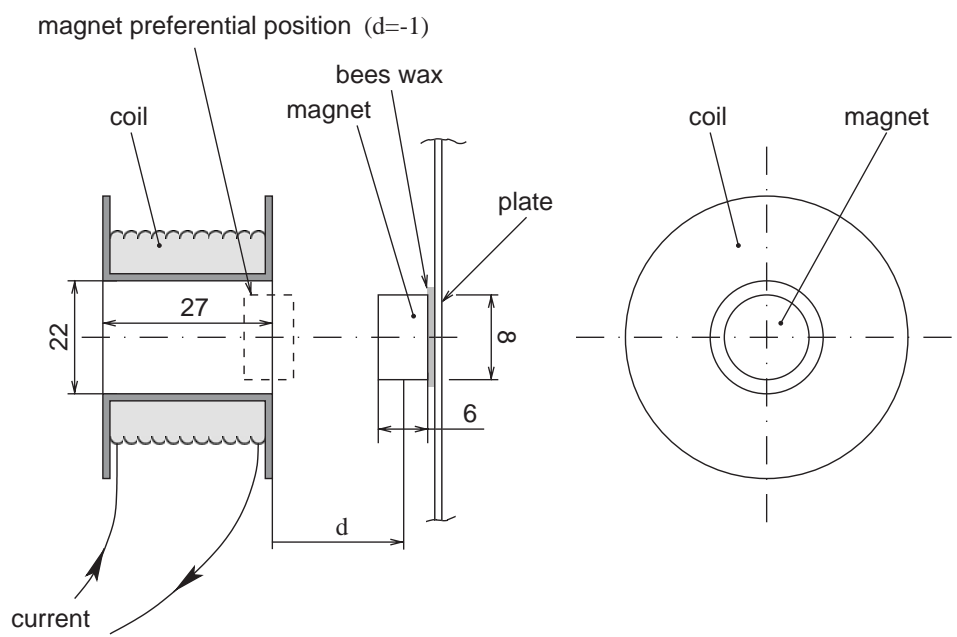

Fig. 2. Electromagnetic exciter. Distance $d$ is measured from the right side of the coil to the middle of the magnet, at rest. The magnet is radially centred in the coil cavity. Dimensions are in $\mathrm{mm}$.

A calibration procedure of the coil/magnet system has been carried out, in order to evaluate the force acting on the magnet, as a function of the intensity of the current in the coil. The details are shown in Appendix A. It has been found that the force is proportional to the intensity, under the 


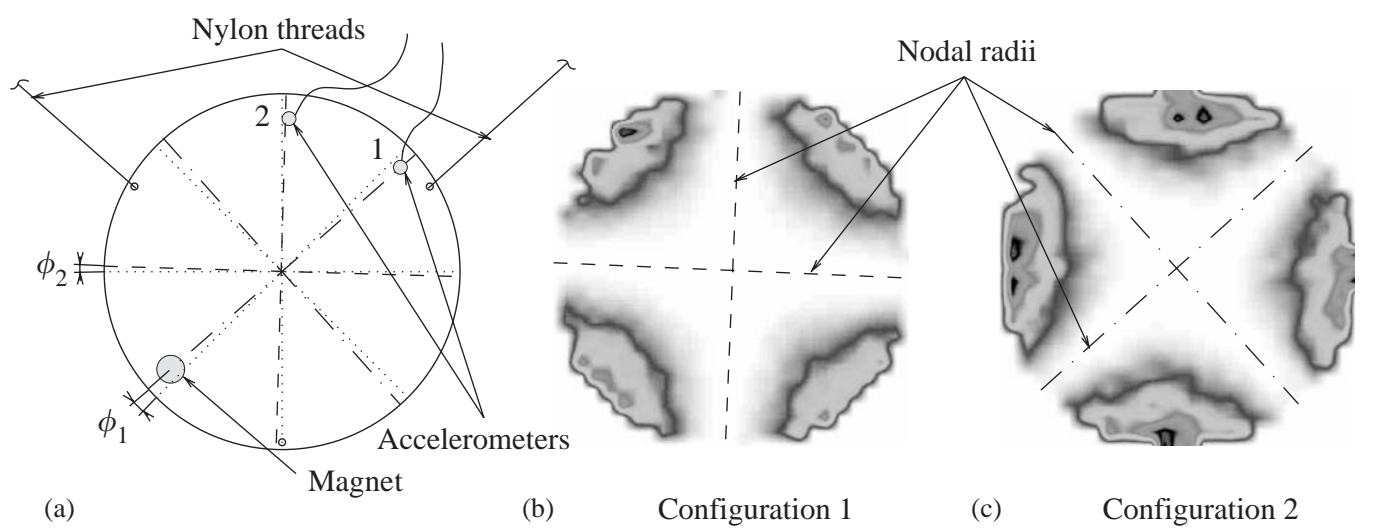

Fig. 3. (a): Fixation of the plate with nylon threads; relative positions of the accelerometers, the magnet and the nodal radii of the two preferential configurations. (b, c): 2-D views of the shapes of the two preferential configurations, resulting from an experimental modal analysis of the plate in situ (the grey zones correspond to the maxima of amplitude, and the white zones to the minima).

condition that the magnet has a constant position with respect to the coil. The proportionality coefficient $K$ depends on the position of the magnet with respect to the coil, and especially to the distance $d$ between the side of the coil and the middle plane of the magnet (Fig. 2). As the magnet follows the plate oscillations during experiments, the force is actually not purely proportional to the intensity. In particular, when the current is sinusoidal, harmonic distortion of the force signal occurs. The distance $d=-1 \mathrm{~mm}$ has been chosen as the position at rest of the magnet, because $K$ is a locally symmetric function around this position. With this choice, the harmonic distortion is the weakest and is composed mainly of odd order components. This position is also the maximum of $K$. The magnet is radially centred on the symmetry axis of the coil.

The proportionality coefficient between the force and the intensity slightly depends on the frequency $\Omega$ of the signal, probably because a fraction of the driving energy is dissipated in the magnet by Foucault's current (Appendix A). This feature has been previously noticed by Kung and Pao [3]. An average value of the ratio between the force and the intensity is $K=0.23 \mathrm{~N} \mathrm{~A}^{-1}$, for frequencies $\Omega / 2 \pi$ between 50 and $200 \mathrm{~Hz}$. This value will be used in the following to estimate the force acting on the plate by measuring the intensity of the current in the coil.

Two accelerometers are used to measure the oscillations of both preferential configurations. Accelerometer 1 (resp. 2) is located on a node of configuration 2 (resp. 1), so that the contribution of both configurations are properly discriminated (Fig. 3). The signals of the accelerometers are sent to a charge amplifier. An oscilloscope and a spectrum analyzer are used to observe the modulation and the FFT spectrum of the two signals. Two multimeters give the root mean square (r.m.s.) value of the signals amplitudes, and an oscilloscope is used to measure the phase between the two oscillations. The phase between configuration 1 and the driving force is measured with another oscilloscope, fed by the terminal voltage of the ammeter. This device is equivalent to a small constant resistance, which implies that its terminal voltage is proportional to the intensity in the coil, and then to the driving force.

When the plate undergoes deflection amplitudes of the order of a millimeter, the signals delivered by the accelerometers are not pure sine waves (of frequency $\Omega$ ), as would be expected for 
a linear system. Harmonic components, of small, but not negligible magnitude compared to the fundamental, often disturb the measurements of amplitude and phase, because the resulting shape of the signals are not perfectly sinusoidal (see for example Fig. 10). It was decided to add a lowpass filter, so that all quantitative measurements are made on the fundamental component of the accelerations. This is justified by the fact that the theoretical curves exhibited in Part I stem from a first order multiple scale development. Hence, the comparison with experimental results are to be done on the fundamental component of the signals. Moreover, the measurements of the phase differences are easier, and the results given by the multimeters are good estimates of the r.m.s. value of the amplitude of the fundamental components (the multimeters do not calculate the real r.m.s. value of the signal, but give a good estimates of it if the signal is perfectly sinusoidal). As a conclusion, the measurements (amplitudes and phases) presented in the following sections will refer to the fundamental component of the signals delivered by the accelerometers.

\subsection{Preferential configurations of the plate}

The plate was hung up by two threads only, in order to avoid an unwanted pre-stretching of the mid-plane of the plate due to the tension in the threads (Fig. 3(a)). The edge of the plate is then assumed to be free, under the assumption that the threads disturb the motion of the edge as little as possible.

The asymmetric mode $(2,0)$ (2 nodal radii, no nodal circles) has been chosen for the experiments of this paper. A preliminary modal analysis of the plate, focused around the two preferential configurations, has been carried out by means of a laser vibrometer (OMETRON, VPI sensor). This study gives the modal shapes of the two preferential configurations, depicted by the views (b,c) of Fig. 3. For a perfect plate with perfectly free boundary conditions, the position of the nodal radii are arbitrary. The presence of the hanging threads sets boundary conditions that are not perfectly free everywhere on the edge, and thus fixes the position of the nodal radii. The addition of the magnet and the accelerometers glued on nodes and/or antinodes does not change radically the nodal pattern. The position of the nodal radii can be measured by the two angles $\phi_{1}$ and $\phi_{2}$, with respect to the vertical symmetry. In particular, the nodal radii of one configuration does not fall exactly midway between the radii of the other. This imperfection is measured by $\left|\phi_{1}-\phi_{2}\right|$. This is a consequence of: (i) the structural imperfections of the plate, (ii) the deviation from the exact vertical symmetry of the nylon threads, and (iii) the position of the added masses glued on the plate. The exact value of the angular shift $\left|\phi_{1}-\phi_{2}\right|$ and angles $\phi_{1}$ and $\phi_{2}$ are of the order of one degree.

The position of the accelerometers and the magnet must be carefully adjusted. This can be carried out in two steps. First, the modal shapes of Figs. 3(b,c) allow one to roughly locate the nodal radii. Secondly, the best position for the accelerometers and the magnet is finely adjusted, so that the signal delivered by accelerometer 2 is as low as possible when the magnet drives configuration 1 . It has been found very difficult to drive only configuration 1 , even if the magnet is precisely located on the nodal radii of configuration 2, mainly because the area of the magnet in contact with the plate is finite.

The natural frequencies $f_{1}$ and $f_{2}$ of the two preferential configurations are slightly different, and depend on the previously enumerated experimental conditions (i, ii and iii). Table 2 gives an example of the variation of the natural frequencies with the presence and location of the added 
Table 2

Measured natural frequencies of both preferential configurations

\begin{tabular}{lll}
\hline & Configuration 1: $f_{1}(\mathrm{~Hz})$ & Configuration 2: $f_{2}(\mathrm{~Hz})$ \\
\hline Hanged "naked" plate & 108 & 111 \\
Both accelerometers & 107.5 & 110.5 \\
Magnet & 106 & 111 \\
Magnet and accelerometers & 105.5 & 110.5 \\
Ballast, magnet and accelerometers & 105.5 & 108 \\
\hline
\end{tabular}

masses. These frequencies were measured by means of the spectrum analyzer, with the plate subjected to free vibrations. The added masses are located as shown in Fig. 3(a). A magnet (of mass $6 \mathrm{~g}$ ) glued on an anti-node of configuration 1, at $1 \mathrm{~cm}$ of the edge, lowers its natural frequency $f_{1}$ by approximately $2 \mathrm{~Hz}$. An accelerometer (of mass $0.6 \mathrm{~g}$ ), at a similar location, lowers the frequency by $0.5 \mathrm{~Hz}$. In order to obtain a difference of natural frequencies that is not too large, a ballast (of mass $11 \mathrm{~g}$ ) can be glued on an anti-node of configuration 2 . It lowers $f_{2}$ by $1.5 \mathrm{~Hz}$.

The variations of nodal patterns and natural frequencies considered above have been previously noticed in Ref. [8] and taken theoretically into account in Ref. [13] and in the model presented in Part I [1].

\subsection{Measuring the resonance curves}

In what follows, experimental resonance curves for both preferential configurations will be presented (Figs. 5-7, 13, 14). The amplitude of the excitation of the plate, denoted by $T_{d r}$, is held fixed, the frequency $\Omega$ is slowly varied up and down around the natural frequencies of both configurations, and the deflections $w_{1}$ and $w_{2}$ of both configurations are plotted. Thus, the signal of the accelerometers must be integrated twice. As explained in Section 2.1, the r.m.s. amplitudes $s_{1}^{(r . m . s .)}$ and $s_{2}^{(r . m . s .)}$ and phases $\varphi_{1}$ and $\varphi_{2}$ of the filtered signals given by the accelerometers are measured. Assuming that those signals are pure sine waves (of frequency $\Omega$ ), the two integrations are equivalent to the following equations:

$$
\begin{gathered}
a_{1}=\frac{\sqrt{2}}{\Omega^{2}} s_{1}^{(r . m . s .)}, \quad a_{2}=\frac{\sqrt{2}}{\Omega^{2}} s_{2}^{(r . m . s .)}, \\
\gamma_{1}=\varphi_{1}+\pi, \quad \gamma_{1}=\varphi_{2}+\pi,
\end{gathered}
$$

where $\left(a_{1}, \gamma_{1}\right)$ and $\left(a_{2}, \gamma_{2}\right)$ are the amplitude and phase with respect to the driving force $T(t)$, of the deflections $w_{1}$ and $w_{2}$ respectively. Their mathematical expressions can be written as

$$
\begin{gathered}
T(t)=T_{d r} \cos \Omega t, \\
w_{1}=a_{1} \cos \left(\Omega t-\gamma_{1}\right), \quad w_{2}=a_{2} \cos \left(\Omega t-\gamma_{2}\right) .
\end{gathered}
$$

In the following, all the numerical results denoting amplitudes (of deflection or forcing) are related to the measured real amplitudes, and not to the r.m.s. ones. 


\section{Non-linear coupling between preferential configurations}

In this section, a typical case of non-linear coupling between the preferential configurations of the plate is considered. Only a one-to-one combination resonance is addressed here. It excludes the excitation of higher order modes, which are likely to be observed for higher forcing than those presented in this paper. This kind of problem is treated for example by Lewandowski [14] with a numerical model.

The magnet and the accelerometers are located as specified in Fig. 3(a). A ballast (of mass $11 \mathrm{~g}$ ) is added on an antinode of configuration 1, in order to lower the difference of natural frequencies. This operation is necessary to obtain the coupling phenomenon between the two configurations for driving forces of magnitude small enough so that the one-to-one combination resonance assumptions are fulfilled (larger forces lead to excitate other modes than the preferential configurations. See Part 1 for the influence of the internal detuning on the instability region). The resulting measured natural frequencies are those of the last row of Table 2. The directly excited configuration is the first one, of natural frequency $f_{1}=105.5 \mathrm{~Hz}$, lower than $f_{2}=108 \mathrm{~Hz}$, the natural frequency of the other configuration. The internal detuning is positive, which leads to $\sigma_{1}>0$ in the model of Part I.

In what follows, the response of the plate is analyzed for two different levels of forcing. As a preliminary, Fig. 4 shows the theoretical single-degree-of-freedom solution (s.d.o.f., see Ref. [1]), for the corresponding level of forcing. One can notice that as the s.d.o.f. curve (denoted by (1)) for the lowest forcing does not enter the instability region, no coupled solution between the two configuration is expected. On the contrary, the s.d.o.f. solution (curve number (3)) for the largest forcing enters into the instability region. Thus, a coupled regime between the two configurations is predicted, leading to an energy transfer from the directly excited configuration (the first one) to the other. Fig. 4 shows in particular that a minimum forcing (curve (2)) is necessary to obtain a coupled solution (see Ref. [1]).

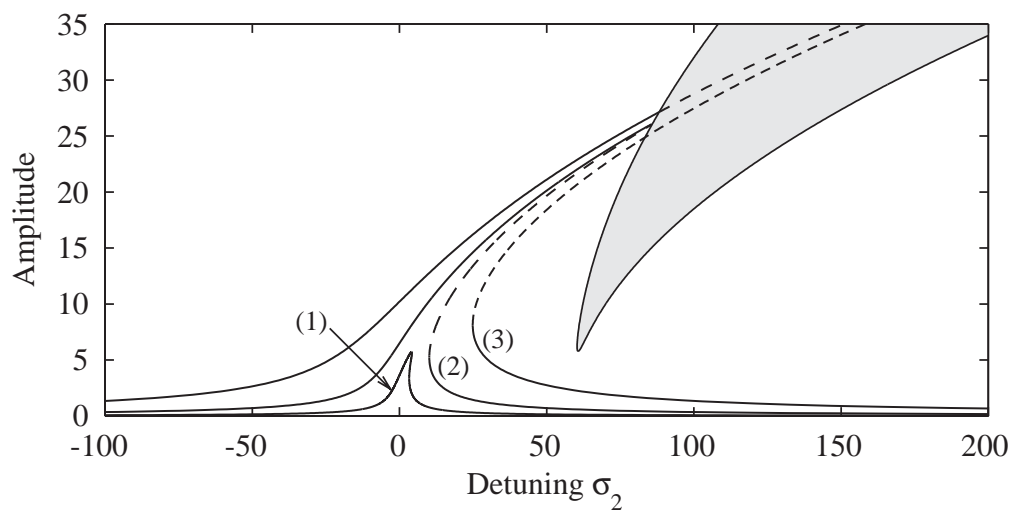

Fig. 4. Theoretical s.d.o.f. solutions and instability region for three different forcing. $(1): T_{d r}=9.9 \times 10^{-3} \mathrm{~N},(2)$ : $56 \times 10^{-3} \mathrm{~N}$ and (3): 0.17 N. (1) and (3) corresponds to the two experimental situations of Figs. 5 and 6 ; (2) corresponds to the minimum forcing magnitude which is necessary to obtain the coupled solution. 


\subsection{Experimental observations}

Fig. 5 shows the amplitudes and phases of $w_{1}$ and $w_{2}$ for a constant forcing amplitude of $9.9 \times 10^{-3} \mathrm{~N}$, corresponding to curve (1) in Fig. 4. Even if the magnet drives mainly configuration 1, a weak resonant excitation of configuration 2 can be noticed around $108 \mathrm{~Hz}$. The responses $w_{1}$ and $w_{2}$ of the two configurations can be denoted as uncoupled, as they exhibit typical resonance curves of two uncoupled forced s.d.o.f. systems [17]. The amplitude $a_{1}\left(a_{2}\right)$ passes through a resonance peak, while the phase $\gamma_{1}\left(\gamma_{2}\right)$ changes from 0 to $\pi \mathrm{rad}$, in a localized forcing frequency range centred on the natural frequencies of configuration $1(2), f_{1}=105.5 \mathrm{~Hz}\left(f_{2}=\right.$ $108 \mathrm{~Hz}$ ). The forcing is enough for $w_{1}$ to exhibit the classical jump phenomena of a weakly nonlinear system, while $w_{2}$ stays in the linear range.
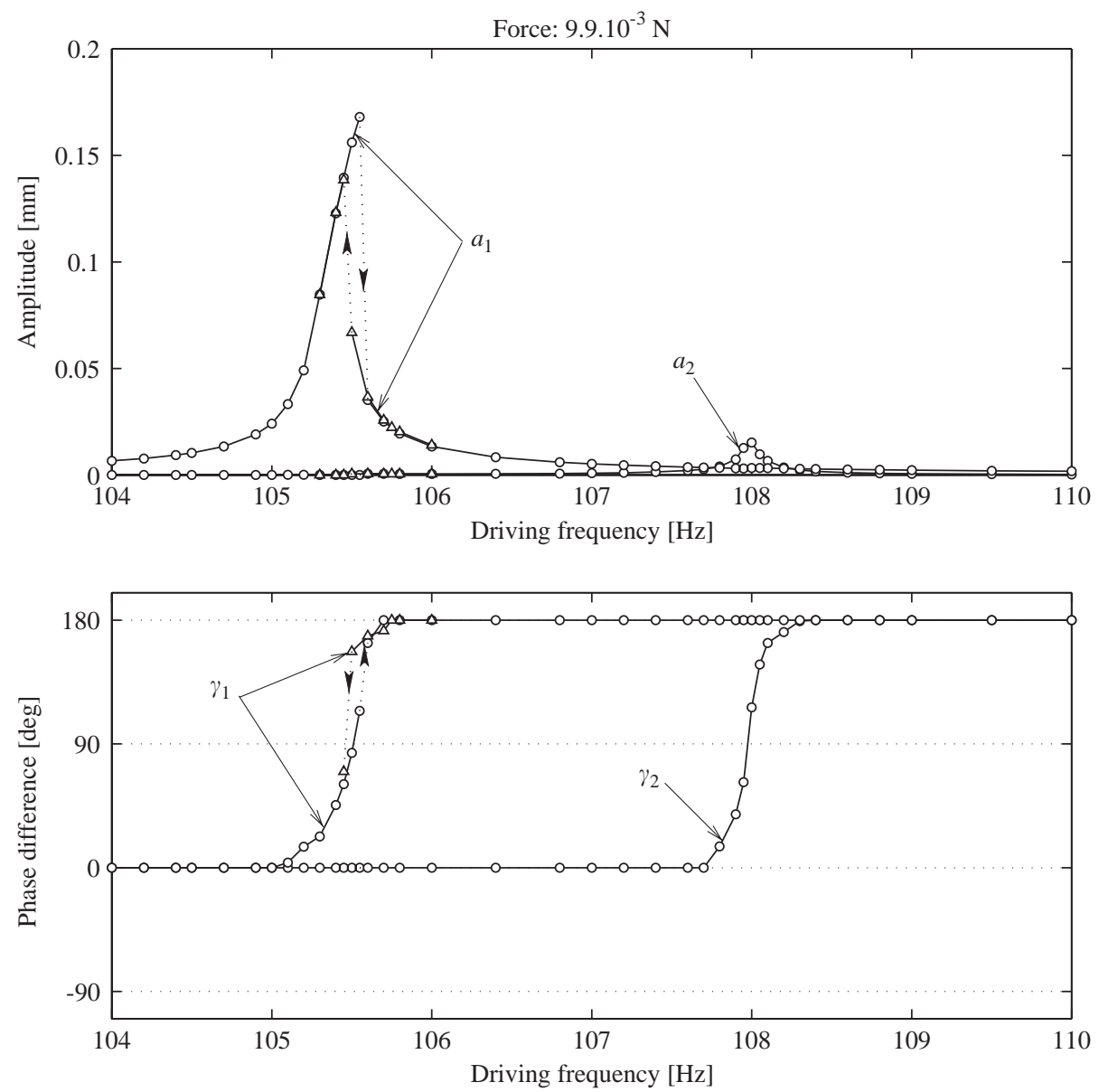

Fig. 5. Experimental resonance curves of the two preferential configurations, for a force amplitude of $9.9 \times 10^{-3} \mathrm{~N}$. $\circ$ : increasing frequencies; $\triangle$ : decreasing frequencies. $\left(w_{1}, \gamma_{1}\right)$ and $\left(w_{2}, \gamma_{2}\right)$ denotes the deflection and the phase with respect to the force, as measured by accelerometers 1 and 2 respectively. 

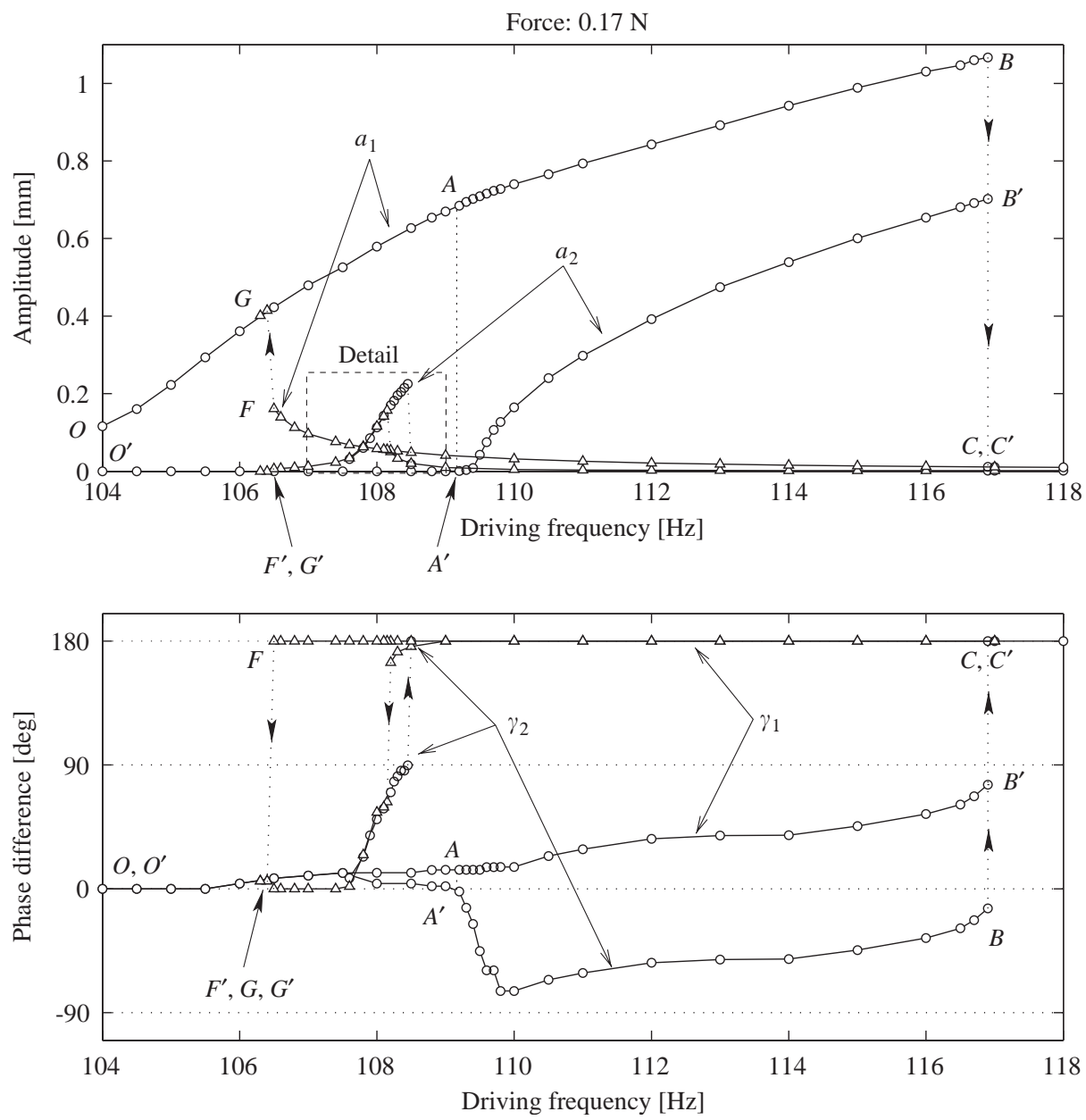

Fig. 6. Experimental resonance curves of the two preferential configurations, for a constant force amplitude of $0.17 \mathrm{~N}$. $\circ$ : increasing frequencies; $\triangle$ : decreasing frequencies. $\left(a_{1}, \gamma_{1}\right)$ and $\left(a_{2}, \gamma_{2}\right)$ denotes the amplitude and phase with respect to the force, of the deflection of configurations 1 and 2.

A similar experiment is reported in Fig. 6. It is conducted with a forcing amplitude of $0.17 \mathrm{~N}$, which correspond to curve (3) in Fig. 4. With this magnitude of forcing, a coupled regime is expected. The experiment will now be described. Starting with a driving frequency of $104 \mathrm{~Hz}$, only one solution for $w_{1}$ and $w_{2}$ is possible, that corresponds to points $O$ and $O^{\prime}$ respectively. This solution will be denoted by $\zeta_{1}$, for which $a_{2}$ nearly equals zero. As the frequency is increased, points $A$ and $A^{\prime}$ are reached, and $a_{2}$ starts to increase. It can be explained by a non-linear coupling between the two configurations, the first (directly excited) one giving energy to the second. From this point, $a_{1}$ and $a_{2}$ increase jointly until they reach points $B$ and $B^{\prime}$. The two deflections $w_{1}$ and $w_{2}$ are nearly in phase, with $\gamma_{1}$ and $\gamma_{2}$ slowly increasing from point $O$ to point $A$. At the beginning of the coupling, $\gamma_{2}$ quickly decreases, so that $w_{2}$ becomes in quadrature of phase with respect to 


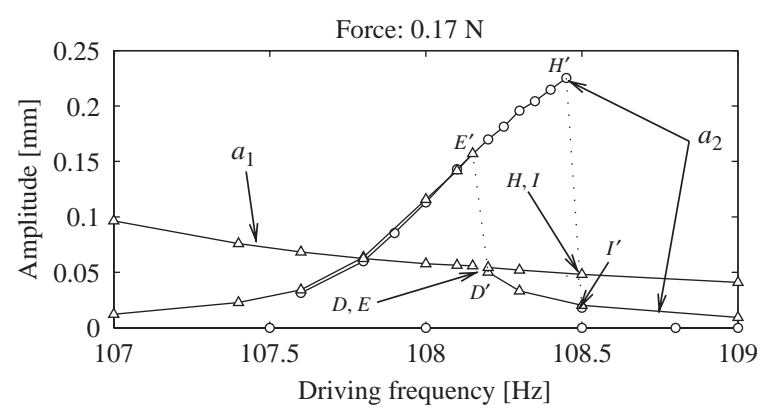

Fig. 7. Detail of the resonance curves of Fig. 6.

$w_{1}$. The quadrature is held during all the coupling stage, until points $B$ and $B^{\prime}$ are reached. As the frequency is increased further, a jump from point $B\left(B^{\prime}\right)$ to point $C\left(C^{\prime}\right)$ occurs, so that $a_{1}\left(a_{2}\right)$ falls down to nearly zero and $\gamma_{1}\left(\gamma_{2}\right)$ jumps to $\pi$ rad. A second solution for $w_{1}$ and $w_{2}$ is then reached, which is denoted by $\zeta_{2}$.

If the experiment is started at a frequency larger than the one of point $C$, only the second solution $\zeta_{2}$ is obtained. The detail of Fig. 6 is shown in Fig. 7. When decreasing the frequency, points $C$ and $C^{\prime}$ are passed, and the solution reaches points $D$ and $D^{\prime}$, with a noticeable increasing of $a_{2}$ as the driving frequency approaches the natural frequency of configuration 2. If the driving frequency is further decreased, a second jump phenomenon occurs, from point $D^{\prime}$, to point $E^{\prime}$. Another solution for $w_{1}$ and $w_{2}$ is reached, and is denoted by $\zeta_{3}$. The jump is mainly noticeable for $a_{2}$ and $\gamma_{2}$, while $a_{1}$ and $\gamma_{1}$ have a nearly continuous variation. At this stage, two ways are possible. First, if the frequency is decreased again, $a_{1}$ slowly increases, points $F$ and $F^{\prime}$ are reached, and another jump phenomenon occurs. The solution gets points $G$ and $G^{\prime}$, and reaches the first solution $\zeta_{1}$. Secondly, if the frequency is increased from point $E^{\prime}, a_{2}$ increases until point $H^{\prime}$ is reached. With a slight increase of frequency, a jump phenomenon occurs, $a_{2}$ decreases to point $I^{\prime}$, and solution $\zeta_{2}$ is reached again.

In the coupled solution range, i.e., between points $A, A^{\prime}$ and $B, B^{\prime}$ of solution $\zeta_{1}$, the phase of the deflection of configuration 1 with respect to the phase of configuration 2 is very close to $\pi / 2$ rad. As mentioned in Part I, the superposition of the motion of the two configurations leads to a travelling wave, rotating anticlockwise. This remarkable feature has been noticed during the experiments by lighting the plate with a stroboscope, driven by a frequency very close to the frequency of the forcing.

It can be noticed that solution $\zeta_{1}$ is somewhat singular, in the sense that it exhibits the coupled phenomenon between the two preferential configurations, $a_{2}$ remaining nearly zero around $f_{2}=108 \mathrm{~Hz}$, with no resonance. On the contrary, solutions $\zeta_{2}$ and $\zeta_{3}$ are very similar to a solution in which $w_{1}$ and $w_{2}$ would be uncoupled, since $w_{1}$ is similar to the lower stable solution of a Duffing oscillator [2], while $w_{2}$ exhibits a typical non-linear resonance in the vicinity of $f_{2}$. These specific features, which were not taken into account in Ref. [1], are due to the residual forcing of the second preferential configuration discussed in the next subsection. 


\subsection{Effect of non-zero $Q_{2}$}

The main difference between the theoretical resonance curves shown in the previous paper [1] and those measured on a real plate is that one cannot ensure that the forcing $Q_{2}$ of the second configuration is strictly equal to zero (see [1, Eq. (31b)]). Hence the study of the dynamical system governing the evolution of the amplitudes and the phases of the response [1, Eq. (40)] has to be completed with a small residual value for $Q_{2}$. This makes the analytical computations quite impossible since $Q_{2} \neq 0$ implies $a_{2} \neq 0$; hence any calculations have to be done in the complete four-dimensional phase space and are generally unmanageable analytically. The solution branches are then computed numerically by means of the DsTool software [15].

Considering a forcing $Q_{2}$ that is small compared to $Q_{1}$ does not affect the qualitative results obtained with $Q_{2}=0$. The instability region still exists, but is no more contained in the plane $\left(a_{2}=0\right)$. As $a_{2} \neq 0$, all the branches of solutions are separated from the s.d.o.f. resonance curve. In particular, the resonance of $a_{2}$, corresponding to the excitation of the s.d.o.f. oscillator

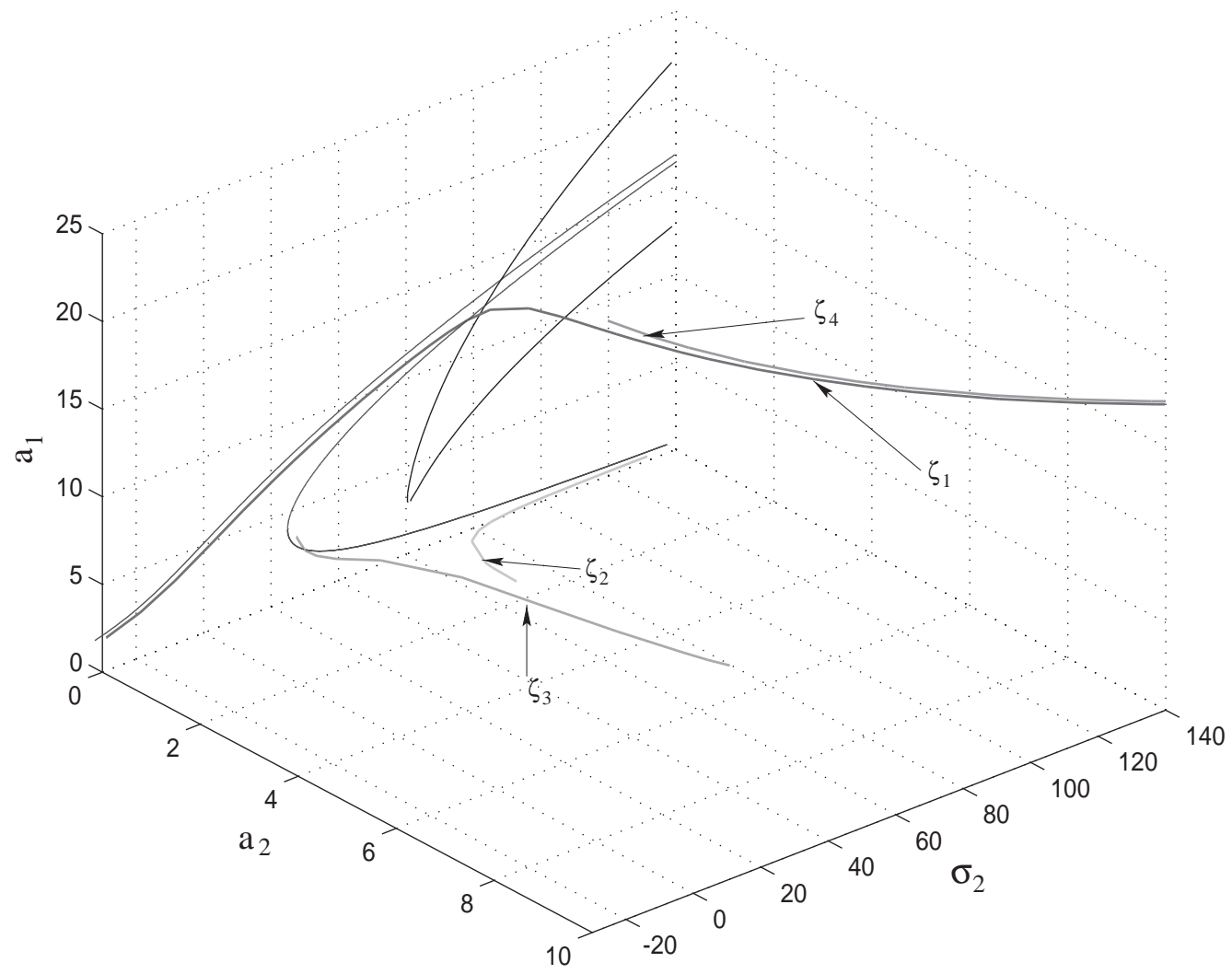

Fig. 8. Theoretical solution branches of the model fitted on the experiments of Fig. 6. The s.d.o.f. resonance curve for $a_{1}$ corresponding to the case $Q_{2}=0$ has been plotted in the plane $a_{2}=0$ to remind its position, although it is not solution branch. The instability region, computed with $Q_{2}=0$, has also been represented. The resonance for $a_{2}$, occurring on the lower branch of $a_{1}$, is clearly visible. The forcing $Q_{2}$ is strong enough to exhibit the non-linearity linked with the second oscillator. 

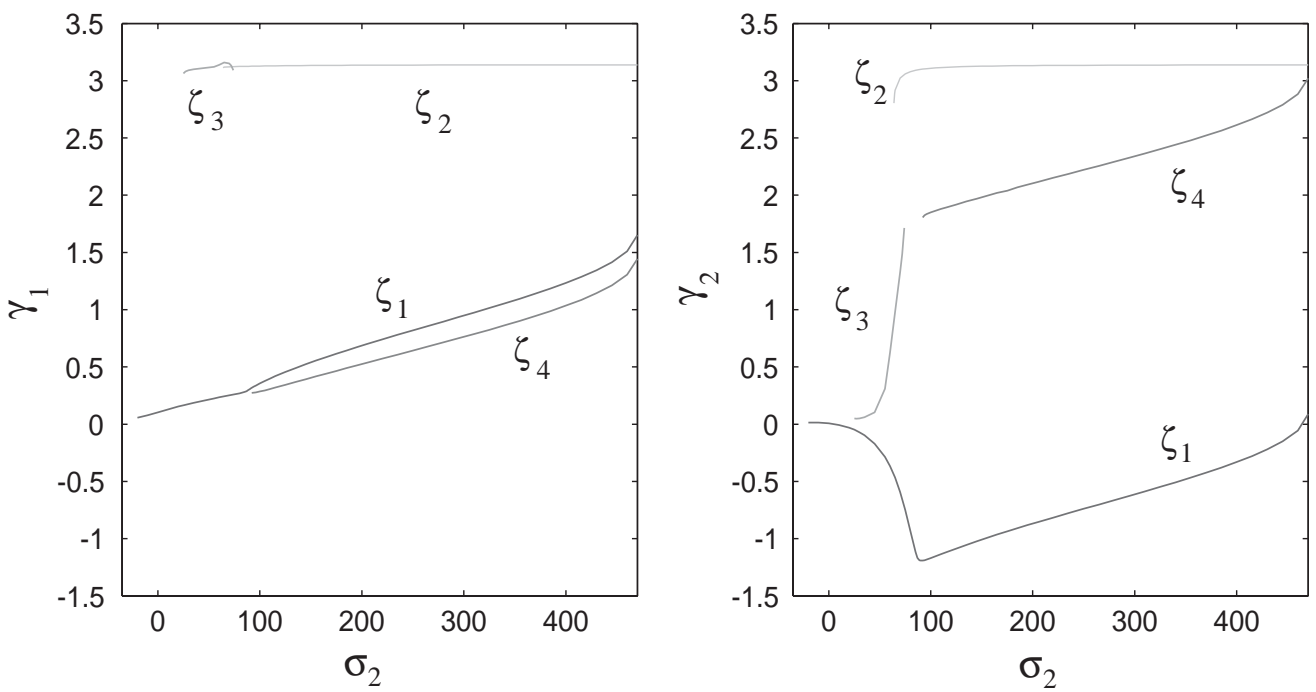

Fig. 9. Theoretical phases of the different branches of Fig. 8: $\gamma_{1}$ on the left and $\gamma_{2}$ on the right. The names of the solution curves have been reported. Unstable states are not reported in this figure. The usual jump phenomenon for $\gamma_{1}$ is clearly visible. The jump phenomenon corresponding to the non-linearity of the second oscillator is visible for $\gamma_{2}$ too (curves $\zeta_{3}$ and $\zeta_{4}$ ). The solution $\zeta_{2}$ displays a phase of nearly $\pi$ with $\zeta_{1}$ for $\gamma_{2}$.

[1, Eq. (31b)] by $Q_{2}$, must correspond to a specific value for $a_{1}$. This occurs for small values of $a_{1}$, and hence is linked to the lower solution branch for $a_{1}$, as can be seen in Fig. 8, where only the stable states have been reported. The branches $\zeta_{2}$ and $\zeta_{3}$ are associated with the resonance for $a_{2}$. This explains why the resonance for $a_{2}$ is experimentally observed only when the excitation frequency $\Omega$ is decreased. When increasing $\Omega$, the upper branch $\zeta_{1}$ is followed, and no resonance occurs for $a_{2}$.

The case considered with the experimental values leads to the appearance of a supplementary branch, denoted $\zeta_{4}$ in Fig. 8. This occurs at $\sigma_{2}=92$ through saddle-node bifurcation. The $\zeta_{4}$ curve is very close to $\zeta_{1}$ for the amplitude values $a_{1}$ and $a_{2}$, but differs from $\zeta_{1}$ by the angle value $\gamma_{2}$. This can be seen in Fig. 9 where the phases with respect to the forcing of the different branches have been represented. One can observe in particular that $\gamma_{1}$ for the $\zeta_{4}$ branch is nearly equal to $\gamma_{1}$ for the $\zeta_{1}$ branch. This is not the case for $\gamma_{2}$ : the two branches exhibits a phase difference nearly equal to $\pi \mathrm{rad}$.

When slowly increasing the excitation frequency $\Omega$, the observed solution is the one given by branch $\zeta_{1}$. Those theoretical curves predict that if a perturbation occurs when $\sigma_{2}>92$, it could be possible for the $a_{2}$ solution to jump from $\zeta_{1}$ to $\zeta_{4}$. This should be visible on the phase $\gamma_{2}$ of the solution. And this has been effectively experimentally observed, by simply knocking on the plate when $a_{2}$ is excited. The time histories of the acceleration of both configurations are shown, for $\zeta_{1}$ and $\zeta_{4}$, in Fig. 10. The positive or negative quadrature clearly appears, and the amplitudes, from one solution to the other, are nearly equals, as it is predicted by the theory (Fig. 8).

Theoretical predictions exhibited by the model have been carefully checked. To conclude with this case, the general fittings are plotted. 

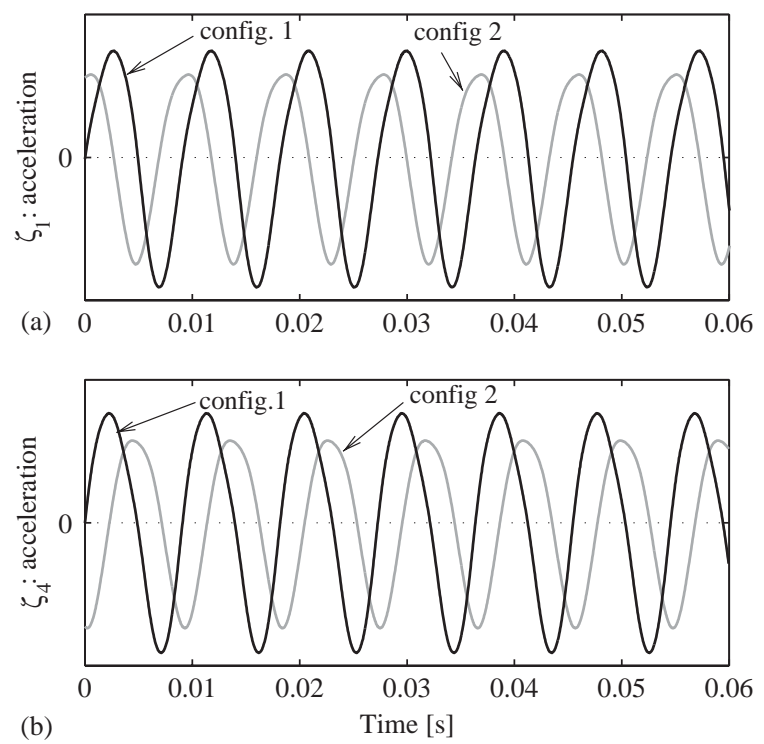

Fig. 10. Measured accelerations, not filtered, of both configurations, for a driving force of frequency $112 \mathrm{~Hz}$ and magnitude $0.17 \mathrm{~N}$, for the two solutions $\zeta_{1}$ (a) and $\zeta_{4}(\mathrm{~b})$.

\subsection{Model fitting to the experiments}

The goal of this subsection is to estimate the parameters of the model so that the theoretical resonance curves fit the experimental ones. Then, the values stemming from this fitting are compared to measurements. It is shown that the two sets of values are of the same order.

The parameters to estimate are defined in [1, Eq. (31)], namely the natural frequencies $\bar{\omega}_{1}$ and $\bar{\omega}_{2}$, the non-linearity coefficient $\Gamma$, the damping coefficient $\bar{\mu}$, and the forcing coefficients $Q_{1}$ and $Q_{2}$. Unlike in Ref. [1], the dimensionless variables are denoted here with overbars, and their relation with the physical variables are given by the following formulae:

$$
\begin{gathered}
r=a \bar{r}, \quad a_{i}=\frac{h^{2}}{a} R_{20}\left(\bar{r}_{\text {mes }}\right) \bar{a}_{i}, \\
\varepsilon=\frac{12\left(1-v^{2}\right) h^{2}}{a^{2}}, \\
f_{i}=\frac{h}{2 \pi a^{2}} \sqrt{\frac{E}{12\left(1-v^{2}\right) \rho}} \bar{\omega}_{i}, \quad f_{d r}=\frac{\Omega}{2 \pi}=\frac{h}{2 \pi a^{2}} \sqrt{\frac{E}{12\left(1-v^{2}\right) \rho}}\left(\bar{\omega}_{1}+\varepsilon \sigma_{2}\right), \\
\mu=\frac{h^{3}}{a^{4}} \sqrt{\frac{12\left(1-v^{2}\right) E}{\rho}} \bar{\mu}, \\
Q_{1}=\frac{a^{5}}{E h^{7}} R_{20}\left(\bar{r}_{d r}\right) T_{d r},
\end{gathered}
$$


Table 3

Physical and model parameters

\begin{tabular}{llll}
\hline & Perfect plate model $^{\mathrm{a}}$ & Measurement $^{\mathrm{b}}$ & Result of fitting $^{-}$ \\
\hline Natural frequencies & $f_{20}=109.2 \mathrm{~Hz}$ & $f_{1}=105.5 \mathrm{~Hz}$ & $\begin{array}{l}f_{1}=105.25 \mathrm{~Hz} \\
\left(\bar{\omega}_{1}=4.9\right)\end{array}$ \\
& & & $\begin{array}{l}f_{2}=108.2 \mathrm{~Hz} \\
\left(\bar{\omega}_{20}=5.09\right)\end{array}$ \\
& - & $f_{2}=108 \mathrm{~Hz}$ & $\left.\bar{\omega}_{d r}=0.17 \mathrm{~N}\right)$ \\
Forcing & & $T_{d r}=0.17 \mathrm{~N}$ & $\left(Q_{1}=1323\right)$ \\
& - & - & $Q_{2} \simeq Q_{1} / 10=150$ \\
Damping & - & $\mu_{1}=0.36 \mathrm{~s}^{-1}$ & $\mu_{1}=0.4 \mathrm{~s}^{-1}$ \\
Non-linearity coefficient & - & $\mu_{2}=0.62 \mathrm{~s}^{-1}$ & $\mu_{2}=\mu_{1}$ \\
& $\Gamma=-1.90$ & - & $\Gamma=-1.65$ \\
\hline
\end{tabular}

${ }^{\mathrm{a}}$ From Ref. [1, Appendices A,B].

${ }^{\mathrm{b}} f_{i}$ and $\mu_{i}$ measured in free vibration, with the ESPRIT method [16]; $T_{d r}$ calculated from the measured intensity (Section 2.1).

where $a_{i}(i=1,2)$ are the amplitudes defined in Eq. (1a), $f_{i}$ denote the natural frequencies in $\mathrm{Hz}, \mu$ is the damping coefficient (in $\mathrm{s}^{-1}$ ) and $T_{d r}$ is the driving force magnitude (in $\mathrm{N}$ ). Moreover, $\bar{r}_{\text {mes }}$ and $\bar{r}_{d r}$ denote the radii of the locations of the accelerometers and driving force respectively. These latter are besides assumed to be glued close to antinodes, so that $w$ and $Q$ depend only on the radial shape $R_{20}$ of mode $(2,0)$ of the plate, defined in Appendix A.1 of Part I.

The parameter estimate is done in two steps. First, the parameters are roughly estimated, either from a theoretical treatment $\left(f_{20}, \Gamma\right)$ described in Appendices A and B of Part I, or derived from measurements $\left(f_{1}, f_{2}, T_{d r}, \mu_{1}, \mu_{2}\right)$. The values of these parameters are summarized in the first two columns of Table 3. Secondly, the parameters are finely adjusted so that the experimental resonance curves fit to the theoretical ones. Fig. 11 shows the result of the fitting, and the third column of Table 3 gives the resulting values of the parameters used in the model fitting.

Now compare all the parameter values, row after row of Table 3. First, the discrepancies between theoretical and measured values of the natural frequencies, due to imperfections of the plate, are discussed in Section 2.2. Secondly, the use of the ESPRIT method [16] shows that both configurations have different damping coefficients, of values $\mu_{1}=0.36 \mathrm{~s}^{-1}$ and $\mu_{2}=0.62 \mathrm{~s}^{-1}$. This feature is not taken into account in the model, in which both configurations have equal damping coefficients. It has been found experimentally that the beeswax used to glue the added masses on the plate tends to damp the vibration. The presence of the ballast at an anti-node of configuration 2 explains probably that $\mu_{2}$ is greater than $\mu_{1}$. Finally, the coefficient $\Gamma$ stemming from the fitting (third column of Table 3 ) is slightly lower (in absolute value) than that calculated from the perfect plate model of Part I (first column). This is explained by all the imperfections of the experimental plate, namely the added masses and the boundary conditions not perfectly free.

Fig. 11 shows that even if the experimental points lie close to the curves resulting from the model, the frequency of the collapsing jump $\left(f_{d r}=117 \mathrm{~Hz}\right)$ is lower than the one predicted by the model $(127 \mathrm{~Hz})$. Using the software DsTool, it has been noticed that the basin of attraction of the present coupled solution (called $\zeta_{1}$ in Section 3.2) is significantly reduced as the driving frequency approaches the collapse point. The step by step increase of frequency from one experimental point 


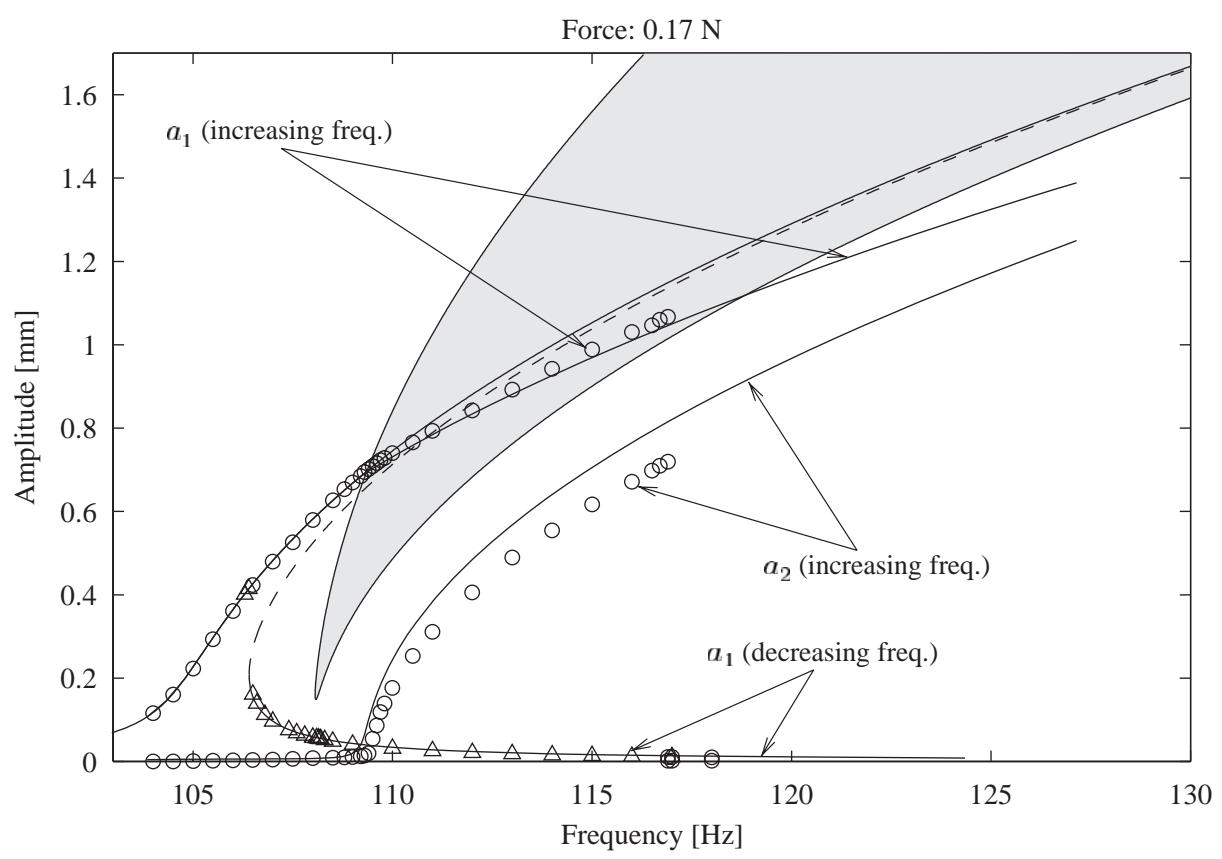

Fig. 11. Fitting of the theoretical model on the experiments. For clarity, the branches for $a_{2}$ obtained with a decreasing frequency (solutions $\zeta_{2}$ and $\zeta_{3}$, shown in Fig. 6) are not plotted. The theoretical solutions are depicted by solid lines and the experimental points by "o" and " $\triangle$ ".

to the next probably yields a perturbation that is sufficient for the solution to leave its basin of attraction, before the collapse frequency predicted by the model is reached. This feature is also a consequence of the fact that the value of $\mu=0.4 \mathrm{~s}^{-1}$ used in the model is lower than $\mu_{2}=0.62 \mathrm{~s}^{-1}$.

As a conclusion, it has been shown through this section that all the qualitative features exhibited during the experiments (jump phenomena, occurrence of the non-linear resonance for $a_{2}$ only in the backward experiment, existence of the $\zeta_{4}$ solution) are predicted by the model. Quantitatively, the differences between the experimental curves and the theoretical ones are rather small.

\section{The case of a negative internal detuning}

\subsection{Theoretical features}

The case of a negative internal detuning has been investigated by simply adjusting the position of the driving magnet at an antinode of the configuration with highest eigenfrequency. Thus, the directly excited configuration is now the second one, whose shape is shown in Fig. 3(c), and whose deflection magnitude is denoted by $a_{2}$. This amounts to consider $\sigma_{1}<0$ in the model.

A specific feature of this case, is that the instability region stays on the left side of the s.d.o.f. resonance curve. Hence, the crossing of the s.d.o.f. resonance curve with the instability region is 


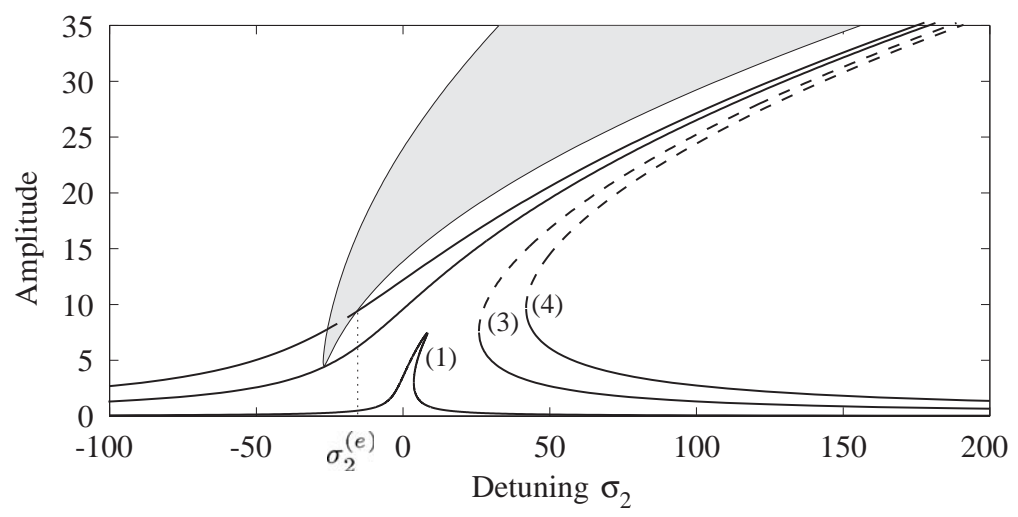

Fig. 12. Theoretical s.d.o.f. solution curves and instability region for three different values of the forcing. (1): $T_{d r}=$ $9.9 \times 10^{-3} \mathrm{~N},(3): 0.17 \mathrm{~N}$ and (4): $0.45 \mathrm{~N}$. Curve (3) corresponds to the same forcing used in the precedent experiment $\left(\sigma_{1}>0\right.$, curve (3) of Figs. 4, 6), and is not sufficient to get the coupled solution. Curve (4) corresponds to the experimental situation of Fig. 13.

only possible for an amplitude of the driving force $T_{d r}$ greater than the one considered in the previous case, $\sigma_{1}>0$, discussed in Section 3. This is shown in Fig. 12, that can be compared to Fig. 4. In particular, curve (3) in Fig. 12 is located just beneath the instability region, whereas it crossed the instability region (curve also denoted (3) in Fig. 4) in the case of a positive internal detuning. Hence no coupled solutions arise. It has been necessary to increase $T_{d r}$ to $0.45 \mathrm{~N}$ (curve (4) in Fig. 12) to observe the coupling between the configurations.

Another important feature is that the s.d.o.f. solution becomes stable again, as it leaves the instability region, as soon as $\sigma_{2}>\sigma_{2}^{(e)}$. Thus, for $\sigma_{2}>\sigma_{2}^{(e)}$, the coexistence of two stables solutions is observable. The coupled solution is obtained by increasing the frequency from $\sigma_{2}<\sigma_{2}^{(e)}$, whereas the s.d.o.f. one can be observed when decreasing the frequency from the lower stable branch, as in a usual s.d.o.f. experiment.

Numerical computations of the coupled solutions in the case investigated here show that the amplitude of the companion configuration $\left(a_{1}\right)$ is greater than that of the excited one $\left(a_{2}\right)$. This fact is experimentally observed (see Fig. 13). A parallel can be established between this case and the internal resonance investigated in Ref. [17], in which the non-excited modes have a greater amplitude than the directly excited one.

Finally, the branch $\zeta_{4}$ depicted in the previous case $\left(\sigma_{1}>0\right)$ for coupled solutions is also present. All those theoretical predictions are confirmed below, in the next subsection.

\subsection{Experimental observations}

Figs. 13 and 14 are similar to Figs. 6 and 7, with a larger forcing amplitude $(0.45 \mathrm{~N})$ and a negative internal detuning. The two natural frequencies are now $f_{1}=107.8 \mathrm{~Hz}$ and $f_{2}=109.2 \mathrm{~Hz}$. The reader must keep in mind that the directly excited configuration is now configuration 2 , whose deflection magnitude is $a_{2}$. As mentioned in the previous subsection, the s.d.o.f. solution is stable for driving frequencies larger than $108.6 \mathrm{~Hz}$, and corresponds to the branch $(J L)$ in Figs. 13 


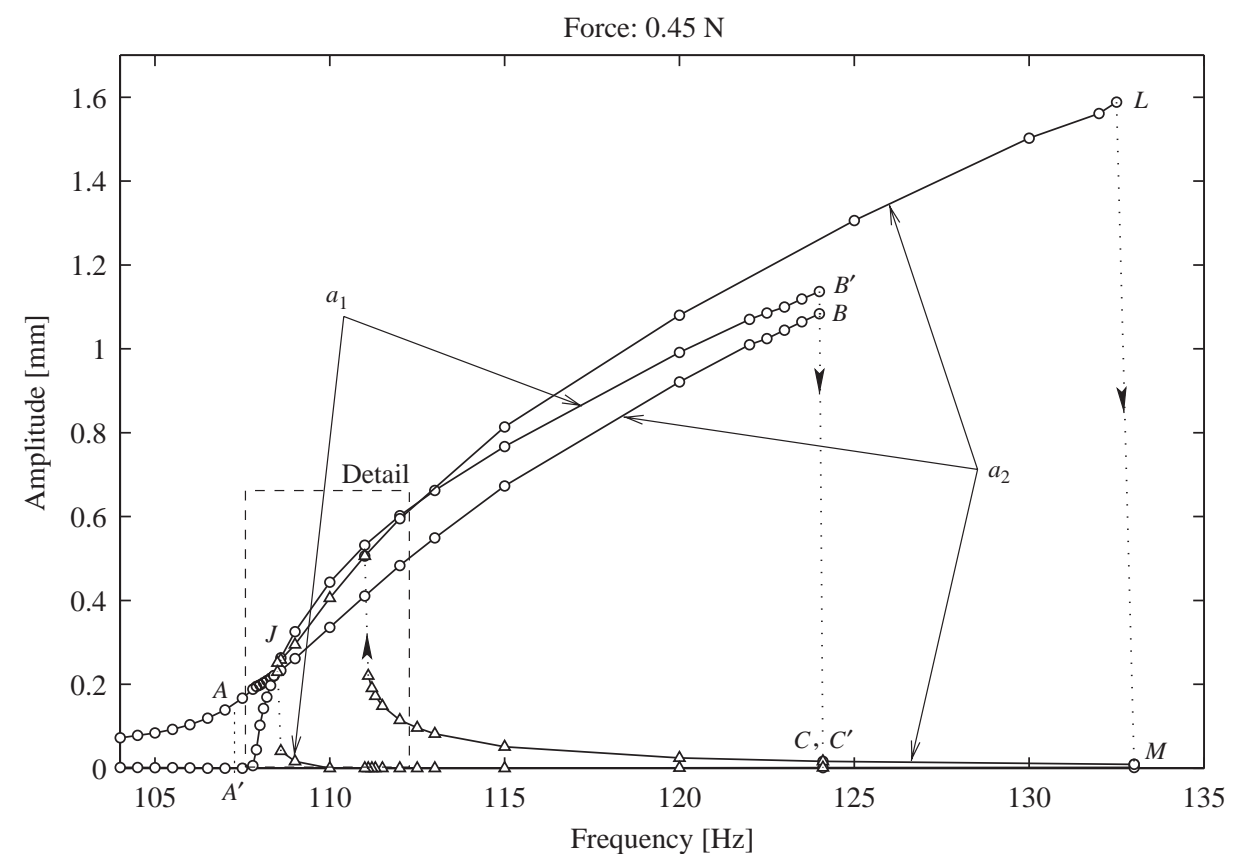

Fig. 13. Experimental resonance curves of the two preferential configurations, for a constant force amplitude of $0.45 \mathrm{~N}$. $\circ$ : increasing frequencies; $\triangle$ : decreasing frequencies. $\left(a_{1}, \gamma_{1}\right)$ and $\left(a_{2}, \gamma_{2}\right)$ denote the amplitude and the phase with respect to the force of the deflections of configurations 1 and 2 .

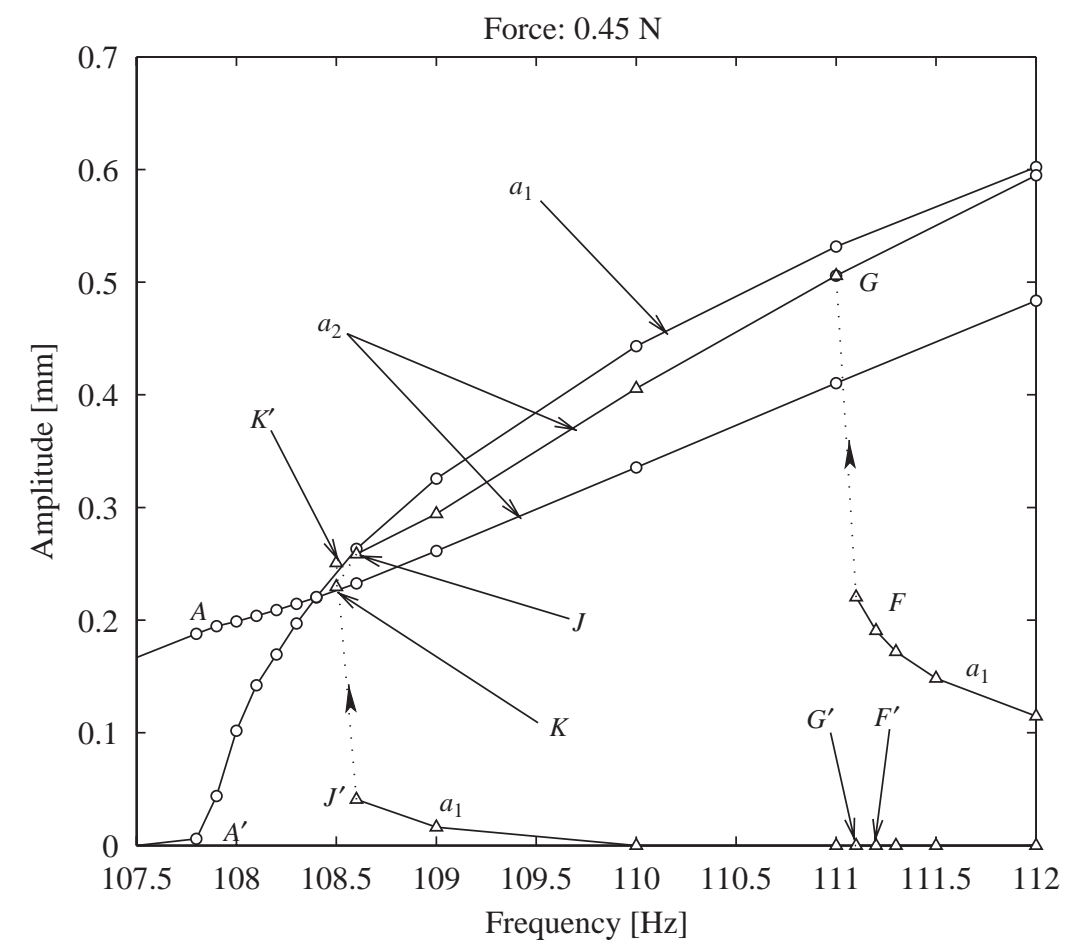

Fig. 14. Detail of the resonance curves of Fig. 13. 
and 14. This solution can be reached after the jump phenomenon from points $F, F^{\prime}$ to points $G, G^{\prime}$. When following this solution by decreasing the driving frequency, points $J, J^{\prime}$ are reached, the s.d.o.f. solution become unstable, and a jump phenomenon occurs. The coupled solution is then reached, by points $K, K^{\prime}$. The special feature of the present case in which the internal detuning is negative is that $a_{1}$ is greater than $a_{2}$. This fact can be observed in Fig. 14. The classical jump phenomenon between the upper and the lower s.d.o.f. solutions is denoted by points $L$ and $M$ for $a_{2}$, whereas $a_{1}$ remains nearly zero.

In addition, it can be noticed that no non-linear resonance occurs for $a_{2}$, in contrast to the case of a positive internal detuning (jump phenomena $D^{\prime} \rightarrow E^{\prime}$ and $H^{\prime} \rightarrow I^{\prime}$ of Fig. 7).

A fitting of the theoretical model of Part $I$ to the present experiments has been found impossible. It can be explained by the fact that for the large forcing considered here, the second order effects cannot be neglected anymore in the model. It will be explained in the next section.

\section{Discussion}

\subsection{Even order harmonic distortion}

In all the experiments carried out on the plate, the presence of a second harmonic (of frequency $2 \Omega$ ) in the acceleration spectrum has been noticed. As the theoretical model involves equations with cubic non-linearities, only harmonics of odd order (of frequencies $3 \Omega, 5 \Omega, \ldots$ ) should have been present. This feature have been previously encountered by Kung and Pao [3], whose measured signals show an asymmetric period shape, which is characteristic of an even components harmonic distortion. Bennouna and White noticed in Ref. [18] and studied in Ref. [19] the presence of a second harmonic in their measurements, and did not find a theoretical explanation for this. A number of conjectures can be made in attempting to explain this phenomenon.

This even component harmonic distortion can be caused first by a loss of symmetry of the vibrating structure in the deflection direction, i.e., normal to the mid-plane of the plate. This normal symmetry, perfect in the case of an ideal plate with perfect boundary conditions, can be broken by (i) the added masses glued only on one side of the plate, (ii) a defect of flatness, and (iii) a defect of symmetry caused by the threads.

Other sources of the presence of even order components have been explored theoretically by Ribeiro in Ref. [20]. This study is devoted to beams, but its conclusions can be extended in the present case, since only cubic non-linearities are involved. This author shows that an even order harmonic distortion is produced if the excitation force (i) is harmonic and not purely transverse, or (ii) if it is transverse harmonic with a constant term.

Finally, the even components present in the measured deflections can be caused directly by an harmonic distortion of the exciting force signal. Section A.3 in Appendix A presents measurements of the harmonic distortion of the force signal as a function of the amplitude of the oscillations of the magnet. It is shown that even if the mean position of the magnet is carefully adjusted at the preferential position $d=-1 \mathrm{~mm}$, an even order harmonic distortion is present in the force signal. For the largest deflections encountered in the measurements of Sections 3 and 4, of order $1.5 \mathrm{~mm}$, the magnitude of the second harmonic component is lower than $-40 \mathrm{~dB}$ with 
respect to the fundamental, which leads to a total harmonic distortion (THD, see Appendix A for the definition) of less than $1 \%$.

A quantitative study is necessary to precisely identify the exact causes of the even component harmonic distortion observed in our measured signals. In particular, the estimation of the even order components magnitude in the deflection of the plate, as a function of the excitation force, is not obvious: a negligible second harmonic of $-40 \mathrm{~dB}$ in the force spectrum is likely to cause a larger distortion in the deflection of the plate. This kind of investigation goes beyond the scope of the present article, as only the fundamental component of the signals has been addressed.

\subsection{Scaling of the measured deflection}

The theoretical model follows from a first order multiple scale analysis (see Section 6 of Part I). It is theoretically valid for a dimensionless deflection $\bar{w}$ of order $O(1)$, only if the small parameter $\varepsilon$ is small compared to 1 (see Section 2.3 of Part I). This involves $w=\left(h^{2} / a\right) \bar{w}$, and the maximal deflection recommended by the theory is then $h^{2} / a=0.023 \mathrm{~mm}$. Nevertheless, Section 3 shows that the model predicts each qualitative feature and that the quantitative features stay in a narrow range, even if the measured deflection is of order $1 \mathrm{~mm}$.

In the case of a much larger forcing of $0.45 \mathrm{~N}$, described in Section 4, even if all the qualitative features are predicted by the model, it has been found difficult to quantitatively fit the model to the experiments. These difficulties can be explained by second-order effects that cannot be neglected for this large magnitude of the driving force. This point is discussed in the next subsection.

As a conclusion, even if the model has been written as a correction to the linear small deflection theory (see Section 2.3 of Part I), it is still valid for a deflection range much larger than that recommended by the mathematics. In particular, the behaviour of the plate is well predicted when it is subjected to deflections $w$ of order of half of its thickness, $h / 2=0.8 \mathrm{~mm}$. This brings a new light on the theoretical work by Nayfeh et al. in Refs. [2,10,17], since the present experiments validate their theory in a larger range of experimental conditions. Nevertheless, a theoretical precise limit of validity of the model is difficult to estimate, although an experimental limit, in terms of the magnitude of driving force, is determined in the next subsection.

\subsection{Second order effects}

Second order effects in perturbative methods can substantially modify the study [21]. The amplitude-frequency relation is first modified [22], which will be discussed below. Secondly, the introduction in the theory requires taking into account the contribution of all the modes that are not excited by the load or by an internal resonance. The result is that the natural linear modes mix with one to another and give non-linear modes, whose shapes are slightly dependent on the deflection amplitude [23]. This effect seems to be significant only at large deflection amplitude [19], larger than the plate thickness, and does not change the quantitative behaviour of the system.

Second order effects appear naturally in the amplitude-frequency relation for the conservative Duffing oscillator. Considering the equation

$$
\ddot{X}+\omega_{0}^{2} X+\varepsilon \Gamma X^{3}=0,
$$


the period $T$ can be calculated by a simple integration in the phase-space trajectory between two turning points (points of maximal elongation where $\dot{X}=0$ ). Let $V(X)$ be the potential derived from Eq. (5), and $E$ the energy of the considered trajectory. Then

$$
T=2 \int_{X_{t}^{(1)}}^{X_{t}^{(2)}} \frac{\mathrm{d} X}{\sqrt{2(E-V(X))}}
$$

where $X_{t}^{(1)}$ and $X_{t}^{(2)}$ are the two turning points [22,24]. Substituting $E$ and $V$ for the appropriate values, the integral is:

$$
T=\frac{2 \pi}{\omega_{0}}\left(1-\varepsilon \frac{3 \Gamma}{8 \omega_{0}^{2}} X_{t}^{2}+\varepsilon^{2} \frac{57 \Gamma^{2}}{256 \omega_{0}^{4}} X_{t}^{4}+O\left(\varepsilon^{2}\right)\right) .
$$

Eq. (7) yields the backbone curve defined by the locus of the peak amplitudes of the resonance curve.

The backbone curve has been experimentally measured in the case of a negative internal detuning $\left(\sigma_{1}<0\right)$, with the values of the natural frequencies of Section 4 . As the upper branch of the s.d.o.f. solution is not completely contained in the instability region, it is possible, when decreasing the frequency, to jump on the upper s.d.o.f. branch. Then increasing the forcing frequency allows one to determine, for a fixed level of the forcing amplitude, the locus $\left(f_{d r}, a_{1}\right)$ of the peak amplitude and hence the backbone curve. This measurement is shown in Fig. 15.

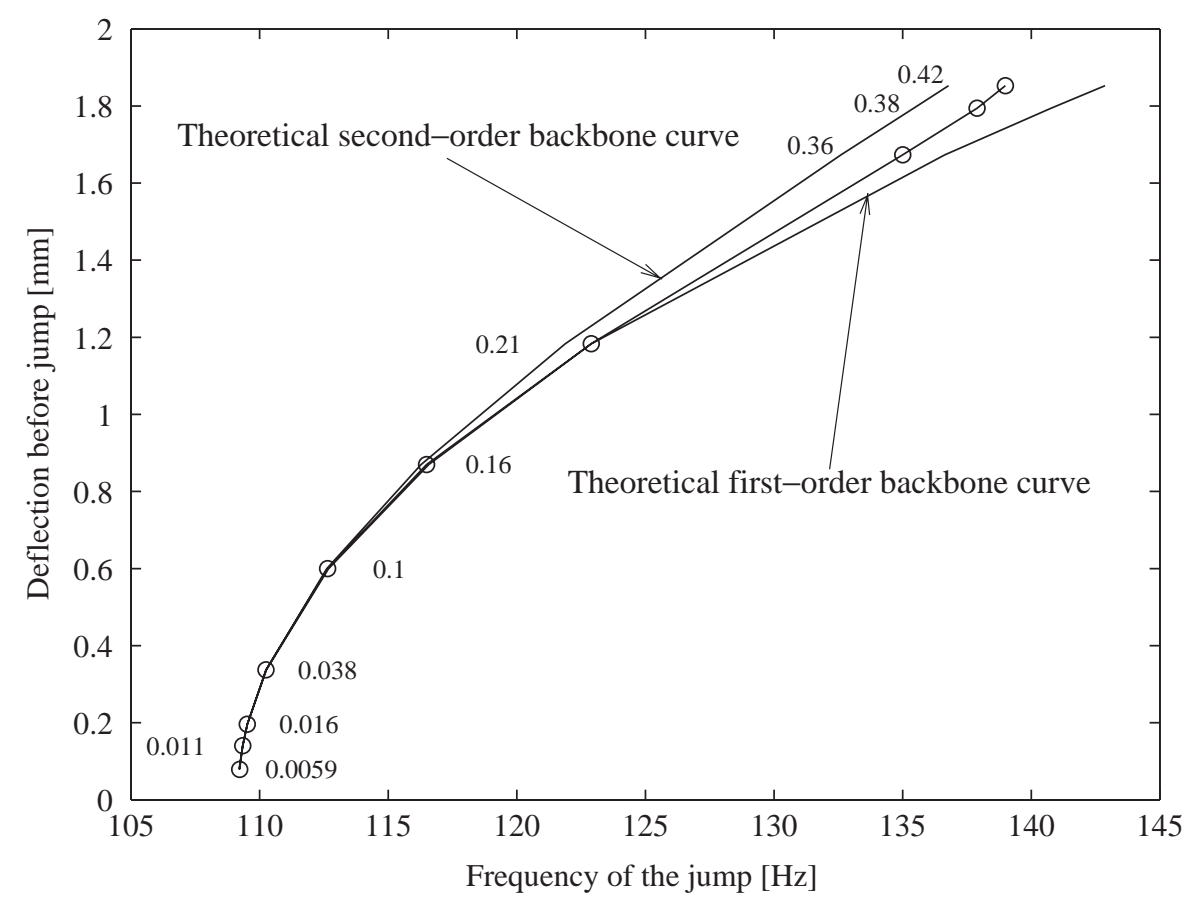

Fig. 15. Experimental backbone curve compared with theoretical ones limited to the first and second order. The driving force magnitude is specified close to the experimental points. The experimental curve has been measured with experimental conditions of Section $4\left(\sigma_{1}<0, f_{1}=105.5 \mathrm{~Hz}, f_{2}=108 \mathrm{~Hz}\right)$. 
In this latter figure, theoretical backbone curves, limited to first order and to second order (according to Eq. (7)), are also presented. These curves can be useful to determine the level of forcing above which second order effects cannot be neglected. Here, one can see that it is the case of a driving force of amplitude greater than $0.16 \mathrm{~N}$, which corresponds to deflections of order half of the plate thickness, $h / 2=0.8 \mathrm{~mm}$. It gives a limit to the validity range of the theoretical model of Part I, and shows that a second order multiple scales development is necessary to quantitatively predict the cases of deflections of order of the plate thickness $h$. In particular, these considerations explain why it has been found difficult to fit the model to the experiments of a forcing amplitude of $0.45 \mathrm{~N}$ discussed in Section 4. However, first order solutions are not completely false in this case since crucial changes such as bifurcations in the phase space due to the second order terms (see Ref. [21]) have not been reached for the level considered in our work.

\section{Conclusion}

In this paper, experimental results are compared to a model developed in Ref. [1]. Both papers provide a complete study of the forced weakly non-linear vibrations of a circular plate, when the deflection is of the order of the thickness of the plate, and when its motion is governed by the two preferential configurations of an asymmetric modal shape only. It is shown that the model predicts each qualitative features exhibited experimentally, such as jump phenomena, resulting from the multivaluedness of the response curves, and a range of driving frequencies in which the motions of the two preferential configurations are coupled. A precise quantitative prediction of the phenomena is obtained when the input parameters of the model are carefully estimated. A method for estimating the parameters is presented. It is based on a fitting of the model to experimental resonances curves.

The limits of validity of the model are discussed. The experiments presented in this paper have shown in particular that the theory of Part $\mathrm{I}$ is valid for a maximum plate deflection of order of half of its thickness $(h / 2)$, which is much larger than the theoretical limitations exposed in Part I and by Nayfeh et al. in Refs. [10,17]. For deflections of order of the plate thickness, it has been shown that second order effects cannot be neglected anymore.

\section{Acknowledgements}

The first author is grateful to the staff of the Laboratoire de Mécanique Physique of University Paris 6, and especially to François Ollivier for his help during the modal analysis. He also wants to thank Jean-Claude Picaud, who has built the experimental system of Appendix A, for his quickness and his know-how. Then, he thanks Bruno Craoued for having passed on to him his long time interest and curiosity in sound phenomena.

\section{Appendix A. Calibration of the electromagnetic exciter}

The amplitude of the force applied to the magnet by the coil depends on the position of the magnet in the non-permanent magnetic field created by the coil. Consequently, a harmonic 
distortion of the force signal appears when the position of the magnet is not constant. A similar feature has been noticed and studied by Tomlinson [25] in the case of an electro-dynamic vibration exciter, although the geometry of this latter is different from our coil/magnet system.

\section{A.1. Apparatus}

In order to examine the characteristics of the electromagnetic exciter used in the present paper, a special device has been built, shown in Fig. 16. It is composed of a moving assembly, free to move in translation with the help of a bearing traveller, in order to minimize friction. Two prestressed springs create a restoring force, so that the system is equivalent to a one-degree-offreedom mass-spring-damper oscillator. The moving assembly is driven by the magnet/coil system under study. An impedance head (Brüel \& Kjær 8001, composed of two piezoelectric transducers), fixed between one end of the moving assembly and the magnet, is used to measure both the acceleration of the moving assembly and the force applied by the magnet. The axial position of the coil can be adjusted with a micrometer screw, not shown in Fig. 16.

As the force transducer is mounted between the magnet and the moving assembly, the measured force $N$ is actually the force $F$ applied by the coil upon the magnet minus the inertia force created by the mass $m$ of the magnet, so that

$$
N=F-m \gamma,
$$

where $\gamma$ is the acceleration of the magnet. In the following experiments, a mass compensation process is used, by subtracting numerically a signal proportional to the signal given by the acceleration transducer of the impedance head to the signal delivered by the force transducer. The force $F$ created by the coil upon the magnet is then properly measured.

\section{A.2. Force amplitude measurements}

The first series of measurements are done with the moving assembly fixed with respect to the coil, by replacing the springs by two fixed length "O" tubes. The idea is to measure the force applied by the coil upon the magnet, without any displacement of the magnet. A similar apparatus

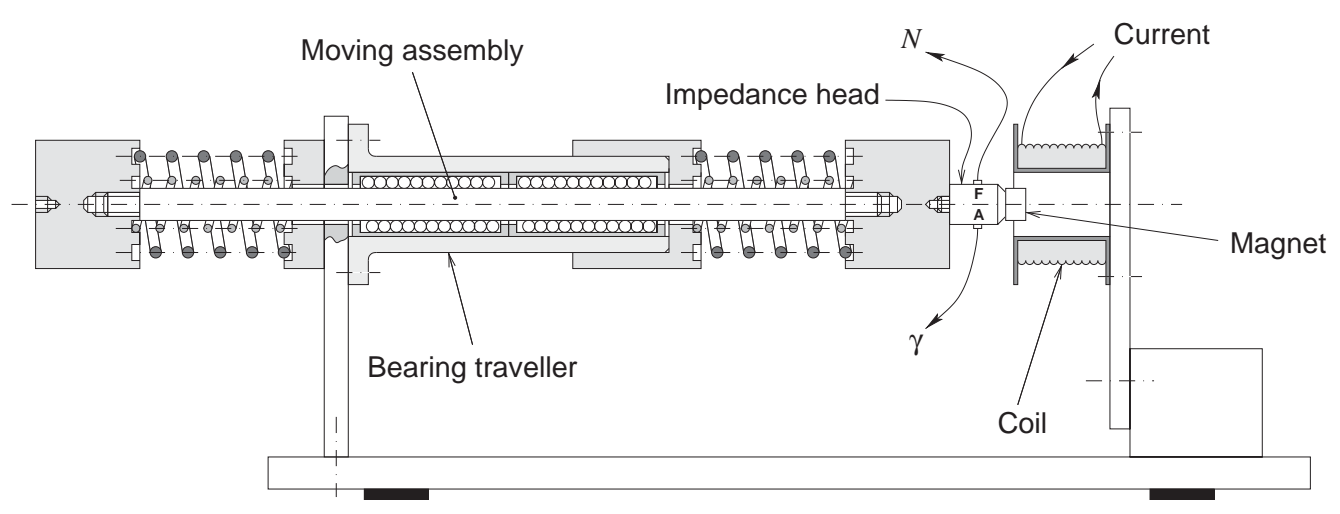

Fig. 16. Sketch of the mass-spring-damper oscillator. 
to the one in Fig. 1 is used for the excitation, with the ammeter measuring the r.m.s. value of the intensity amplitude of the current in the coil.

A sinusoidal current is sent to the coil, and the force created on the magnet is measured. The radial position of the magnet is carefully adjusted in the middle of the coil cavity, as shown in Fig. 2 (left). The different positions of the magnet are marked by the distance $d$ between the side face of the coil and the middle plane of the magnet (Fig. 2). First, the linearity between intensity and force is checked by comparing the two corresponding Fourier spectra. With a perfect sinusoidal current, no harmonics have been noticed in the force signal. Secondly, the proportionality coefficient $K$ between the force and the intensity is calculated from the measured amplitude of the intensity and the force signals (read respectively on the ammeter and a voltmeter, fed by the signal of the force transducer):

$$
\begin{gathered}
i(t)=I_{m} \cos \Omega t, \quad F(t)=F_{m} \cos \Omega t, \\
K=\frac{F_{m}}{I_{m}} .
\end{gathered}
$$

The dependence of $K$ on the position $d$ of the magnet is shown in Fig. 17. One can notice that around the position $d=-1 \mathrm{~mm}, K$ is a symmetric (even) function of $d$. Consequently, only an odd order component harmonic distortion is expected [26]. This is also the position where $K$ is maximum. For these two reasons, $d=-1 \mathrm{~mm}$ is the preferential mean position that has been used in the measurements of this article (see Section 2.1).

In order to estimate the variations of $K$ as a function of the frequency $\Omega$ of the excitation signal, a low-pass filtered white-noise signal is sent to the coil. The Fourier spectrum of $K$ is obtained from the transfer function between force and intensity. The intensity is estimated by measuring the terminal voltage of the ammeter, which is equivalent to a small resistance. Fig. 18 shows $K$ as a function of the frequency, for $\Omega / 2 \pi \in[1 ; 200] \mathrm{Hz}$. One can see that $K$ slightly depends on the

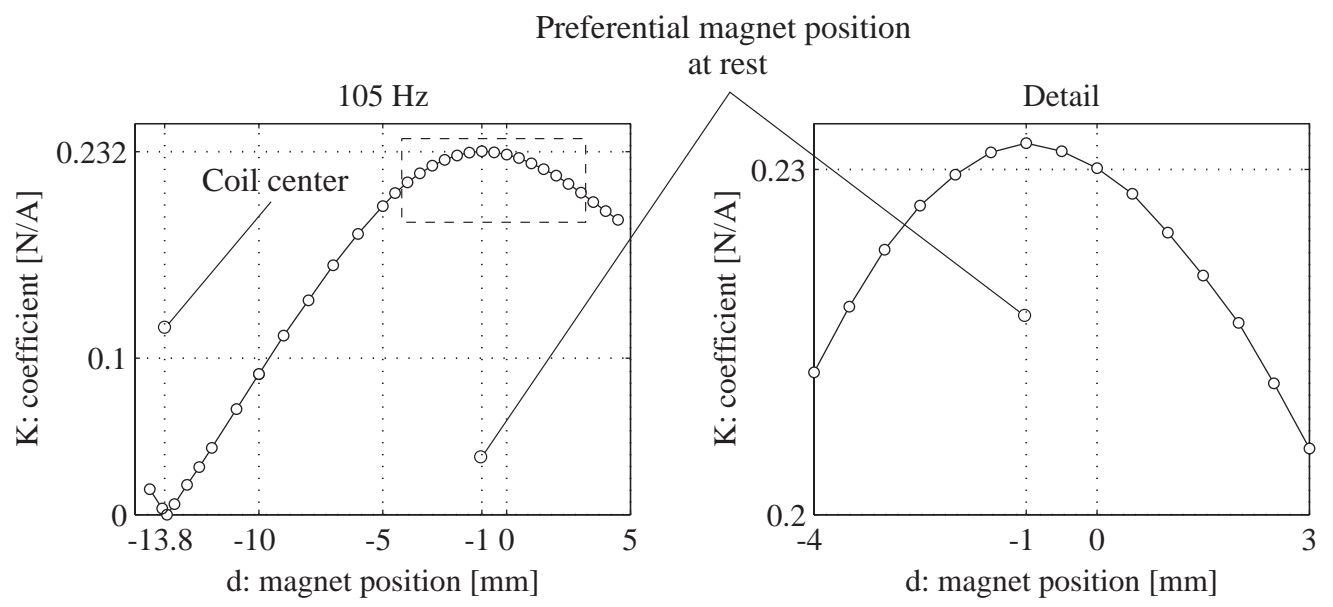

Fig. 17. Proportionality coefficient $K$ between the force and the intensity of the current in the coil, as a function of the fixed position $d$ of the magnet, for a harmonic signal of frequency $\Omega / 2 \pi=105 \mathrm{~Hz}$. 


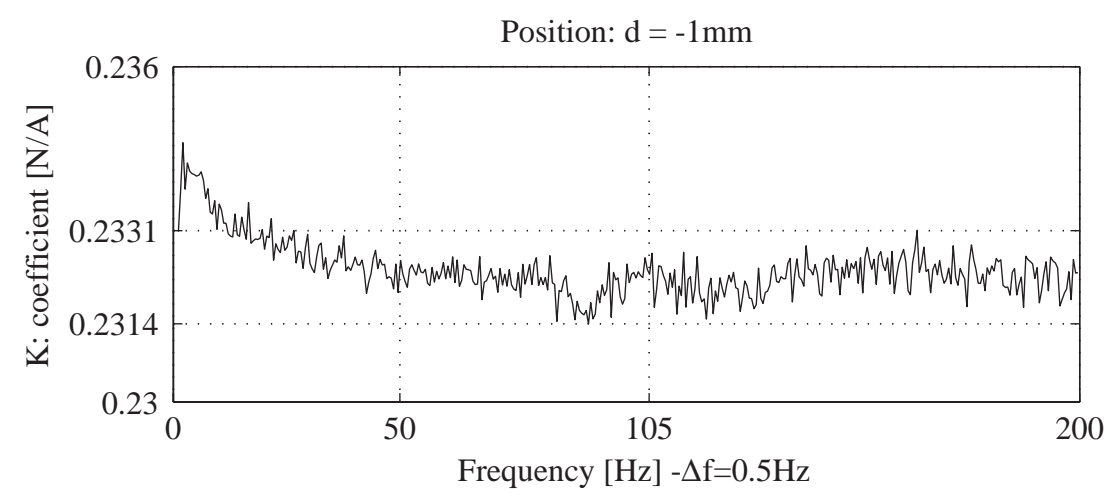

Fig. 18. Proportionality coefficient $K$ between the force and the intensity of the current in the coil, as a function of the frequency of the signal, for the preferential mean position of the magnet $(d=-1 \mathrm{~mm})$.

frequency, probably because of the presence of Foucault's currents in the magnet that bring dissipations. A mean value for $K$ is $0.23 \mathrm{~N} \mathrm{~A}^{-1}$ around $\Omega / 2 \pi=100 \mathrm{~Hz}$, which is the frequency range used in the present article.

\section{A.3. Force distortion}

The aim of the present second series of experiments is to estimate the harmonic distortion of the force, as a function of the amplitude of the oscillations of the magnet. The system of Fig. 16 is now used with the springs, and free to oscillate. A sinusoidal signal of frequency $\Omega$ is sent to the coil, and the force created on the magnet is measured. The amplitude of each harmonic component $H_{n}$ of order $n$ is estimated with the modulus of the FFT of the force at the frequencies $n \Omega(n \in\{1 \ldots 4\})$. It can be noticed that the frequency $\Omega$ must meet one of the discrete frequencies of the FFT. The amplitude of the oscillations of the magnet is obtained by integrating twice the acceleration.

In order to obtain significant amplitudes of vibration of the magnet (up to $6 \mathrm{~mm}$ ), frequency $\Omega$ is chosen as close as possible to the resonant frequency $\Omega_{r}$ of the mass-spring-damper system. For the same reason, the stiffnesses of the springs are chosen so that $\Omega_{r}$ is sufficiently low (for a massspring-damper system, if the mass, the damping constant and the amplitude of the external force are fixed, the lower the stiffness is, the larger the amplitude of the oscillations are, at resonance). The experiments presented in the following have been done with $\Omega_{r} / 2 \pi \simeq \Omega / 2 \pi=21.25 \mathrm{~Hz}$, so that the maximum amplitude of the oscillations obtained without distortion of the amplifier output voltage is $6 \mathrm{~mm}$.

The amplitude $a_{n}$ of each harmonic $H_{n}$ has been measured, as a function of the amplitude of the oscillations of the magnet, for two different mean positions $(d=-1$ and $0 \mathrm{~mm})$. Different amplitudes of the oscillations are obtained by varying the amplitude of the current in the coil. Fig. 19 shows $a_{n}$ in $\mathrm{dB}$, with respect to $a_{1}$, the amplitude of the fundamental component:

$$
a_{n}[\mathrm{~dB}]=20 \log \frac{a_{n}}{a_{1}} .
$$



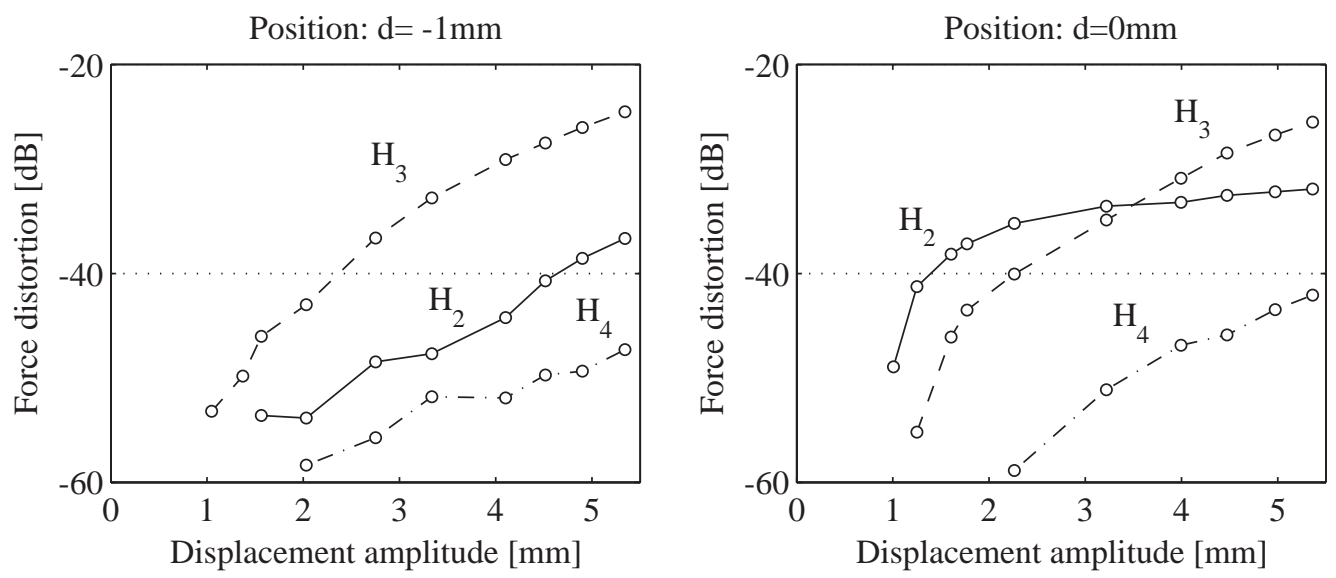

Fig. 19. Amplitude of the first four harmonic components as a function of the oscillation amplitude of the magnet, in $\mathrm{dB}$ with respect to the fundamental component.

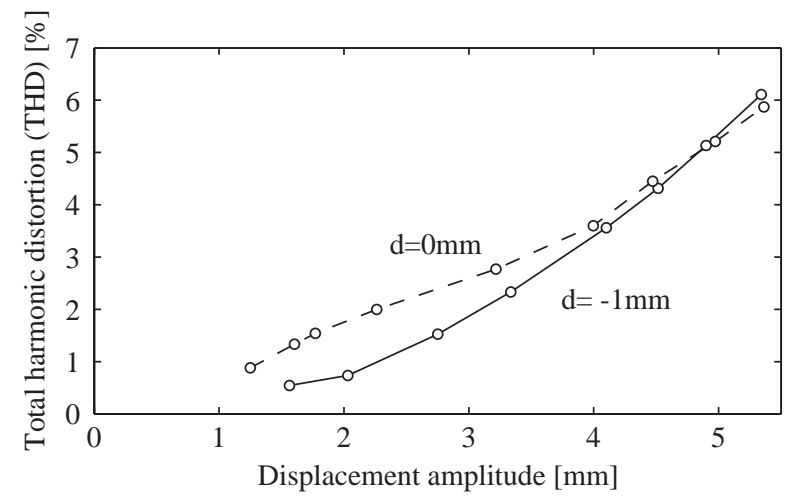

Fig. 20. Total harmonic distortion as a function of the oscillation amplitude of the magnet.

One can see that, as expected, the harmonic content of the force tends to be odd around the mean magnet position $d=-1 \mathrm{~mm}$, because of the symmetry of the curve $K=f(d)$ (Fig. 17) around this position. On the contrary, the force has a full harmonic content around $d=0 \mathrm{~mm}$.

The total harmonic distortion (THD) has also been calculated, by the following formula (from Ref. [26]):

$$
\mathrm{THD}=100 \frac{\sqrt{a_{2}^{2}+\cdots+a_{N}^{2}}}{\sqrt{a_{1}^{2}+a_{2}^{2}+\cdots+a_{N}^{2}}}
$$

It is shown in Fig. 20.

This leads to the conclusion that the position $d=-1 \mathrm{~mm}$ corresponds to the least harmonic distortion and that the mean position of the magnet must be carefully adjusted, in order to 
minimize the harmonic distortion. Moreover, for the amplitudes of the oscillations of the magnet encountered in the experiments of Sections 3 and 4, the amplitude of the harmonic components is less than $-40 \mathrm{~dB}$, corresponding to a THD less than $1 \%$.

\section{Appendix B. Nomenclature}

\begin{tabular}{|c|c|}
\hline & radius of the plate \\
\hline$h$ & thickness of the plate \\
\hline & density of the plate \\
\hline & Poisson ratio \\
\hline$E$ & Young's modulus \\
\hline$f_{1}, f_{2}$ & natural frequencies of the two configurations \\
\hline$\phi_{1}, \phi_{2}$ & angular positions of the nodal radii of the two configurations \\
\hline $\bar{\omega}_{1}, \bar{\omega}_{2}$ & dimensionless angular frequencies of the two configurations \\
\hline$\sigma_{1}$ & nternal detuning between the two preferential configurations \\
\hline$\mu_{1}, \mu_{2}$ & lamping coefficients of the two configurations \\
\hline $\bar{\mu}_{1}, \bar{\mu}_{2}$ & dimensionless damping coefficients of the two configurations \\
\hline & distance between the side of the coil and the middle plane of the magnet \\
\hline$K$ & proportionality coefficient between force and intensity \\
\hline$T(t), T_{d r}$ & signal and amplitude of the exciting force \\
\hline$\Omega, f_{d r}$ & angular frequency and frequency of the force signal \\
\hline$\sigma_{2}$ & $\begin{array}{l}\text { detuning between the excitation angular frequency and the pulsation of the first } \\
\text { configuration }\end{array}$ \\
\hline$Q_{1}, Q_{2}$ & orcing coefficients of the two configurations \\
\hline & imensionless radial position of the forcing \\
\hline$R_{20}$ & theoretical radial shape of the mode $(2,0)$ of a free-edge circular plate \\
\hline$w_{1}, w_{2}$ & deflections of the two configurations \\
\hline$a_{1}, a_{2}$ & deflection amplitudes of the two configurations \\
\hline $\bar{a}_{1}, \bar{a}_{2}$ & limensionless deflection amplitudes of the two configurations \\
\hline$\gamma_{1}, \gamma_{2}{ }_{(r . m . s .)} \quad(r . m . s)$. & deflection phases of the two configurations, with respect to the force signal \\
\hline $\begin{array}{l}s_{1}, S_{2} \\
\varphi_{1}, \varphi_{2} \\
\bar{r}_{\text {mes }} \\
\zeta_{1}, \zeta_{2}, \zeta_{3}, \zeta_{4} \\
\varepsilon\end{array}$ & $\begin{array}{l}\text { acceleration phases of the two configurations, with respect to the force signal } \\
\text { dimensionless radial position of the accelerometers } \\
\text { solution branches } \\
\text { dimensionless small parameter }\end{array}$ \\
\hline & efficient of the cubic non-linear terms in the theoretical model of Part I \\
\hline
\end{tabular}

\section{References}

[1] C. Touzé, O. Thomas, A. Chaigne, Asymmetric non-linear forced vibrations of free-edge circular plates, Part I: theory, Journal of Sound and Vibration 258 (4) (2002) 649-676. 
[2] S. Sridhar, D.T. Mook, A.H. Nayfeh, Nonlinear resonances in the forced responses of plates, Part I: symmetric responses of circular plates, Journal of Sound and Vibration 41 (3) (1975) 359-373.

[3] G.C. Kung, Y.H. Pao, Nonlinear flexural vibration of a clamped circular plate, Journal of Applied Mechanics 39 (1972) $1050-1054$.

[4] K. Yasuda, N. Hayashi, Subharmonic oscillations of a prestressed circular plate, Bulletin of the JSME 25 (202) (1982) 620-630.

[5] O. Thomas, C. Touzé, A. Chaigne, Non-linear resonances in large-deflection vibrations of free-edge circular plates, Proceedings of the Seventh International Congress on Sound and Vibration, 2000.

[6] S.A. Tobias, Non-linear forced vibrations of circular discs, an experimental investigation, Engineering 186 (1958) $51-56$.

[7] C.J.H. Williams, S.A. Tobias, Forced undamped non-linear vibrations of imperfect circular discs, Journal of Mechanical Engineering Science 5 (1963) 325-335.

[8] S.A. Tobias, R.N. Arnold, The influence of dynamical imperfection on the vibration of rotating disks, Proceedings of the Institution of Mechanical Engineers 171 (1957) 669-690.

[9] T.A. Nayfeh, A.F. Vakakis, Subharmonic travelling waves in a geometrically non-linear circular plate, International Journal of Non-Linear Mechanics 29 (2) (1994) 233-245.

[10] S. Sridhar, D.T. Mook, A.H. Nayfeh, Nonlinear resonances in the forced responses of plates, Part II: asymmetric responses of circular plates, Journal of Sound and Vibration 59 (2) (1978) 159-170.

[11] K. Yasuda, T. Asano, Nonlinear forced oscillations of a rectangular membrane with degenerated modes, Bulletin of JSME 29 (255) (1986) 3090-3095.

[12] A. Raman, C.D. Mote Jr., Experimental studies on the non-linear oscillations of imperfect circular disks spinning near critical speed, International Journal of Non-linear Mechanics 36 (2001) 291-305.

[13] G.J. Efstathiades, A new approach to the large-deflection vibrations of imperfect circular disks using Galerkin's procedure, Journal of Sound and Vibration 16 (2) (1971) 231-253.

[14] R. Lewandowski, Computational formulation for periodic vibration of geometrically non-linear structures — Part 2: numerical strategy and examples, International Journal of Solid Structures 34 (15) (1997) 1949-1964.

[15] J. Guckenheimer, M.R. Myers, F.J. Wicklin, P.A. Worfolk, DsTool: a dynamical system toolkit with an interactive graphical interface, Technical Report, Center for Applied Mathematics, Cornell University, Ithaca, NY, 1995.

[16] J. Laroche, The use of matrix-pencil method for the spectrum analysis of musical signals, Journal of the Acoustical Society of America 94 (4) (1993) 1958-1965.

[17] A.H. Nayfeh, D.T. Mook, Nonlinear Oscillations, Wiley, New York, 1979.

[18] M.M.K. Bennouna, R.G. White, The effects of large vibration amplitudes on the fundamental mode shape of a clamped-clamped uniform beam, Journal of Sound and Vibration 96 (3) (1984) 309-331.

[19] R. Benamar, M.M.K. Bennouna, R.G. White, The effects of large vibration amplitudes on the mode shapes and natural frequencies of thin elastic structures. Part III: fully clamped rectangular isotropic plates-measurements of the mode shape amplitude dependence and the spatial distribution of harmonic distortion, Journal of Sound and Vibration 175 (3) (1994) 377-395.

[20] P. Ribeiro, The second harmonic and the validity of Duffing's equation for vibration of beams with large displacements, Computers and Structures 79 (2001) 107-117.

[21] A.H. Nayfeh, N.E. Sanchez, Bifurcations in a forced softening Duffing oscillator, International Journal of Nonlinear Mechanics 24 (6) (1989) 483-497.

[22] A.H. Nayfeh, Perturbation Methods, Wiley, New York, 1973.

[23] A.H. Nayfeh, S.A. Nayfeh, On non-linear modes of continuous systems, Transactions of the American Society of Mechanical Engineers, Journal of Vibration and Acoustics 116 (1994) 129-136.

[24] P. Manneville, Dissipative Structures and Weak Turbulence, Academic Press, New York, 1990.

[25] G.R. Tomlinson, Force distortion in resonance testing of structures with electro-dynamic vibration exciters, Journal of Sound and Vibration 63 (3) (1979) 337-350.

[26] S. Temme, Audio distortion measurements, Brüel \& Kjær Application Note, BO0385-12, 1992. 